\title{
ANALYTICAL THEORY FOR THE INITIAL MASS FUNCTION. III. TIME DEPENDENCE AND STAR FORMATION RATE
}

\author{
Patrick Hennebelle ${ }^{1,2}$ and Gilles Chabrier $3,4,5$ \\ ${ }^{1}$ Laboratoire AIM, Paris-Saclay, CEA/IRFU/SAp-CNRS-Université Paris Diderot, F-91191 Gif-sur-Yvette Cedex, France \\ ${ }^{2}$ Laboratoire de radioastronomie, UMR CNRS 8112, École normale supérieure et Observatoire de Paris, 24 rue Lhomond, F-75231 Paris Cedex 05, France \\ ${ }^{3}$ École normale supérieure de Lyon, CRAL, UMR CNRS 5574, F-69364 Lyon Cedex 07, France \\ ${ }^{4}$ School of Physics, University of Exeter, Exeter EX4 4QL, UK \\ Received 2013 January 11; accepted 2013 April 30; published 2013 June 6
}

\begin{abstract}
The present paper extends our previous theory of the stellar initial mass function (IMF) by including time dependence and by including the impact of the magnetic field. The predicted mass spectra are similar to the time-independent ones with slightly shallower slopes at large masses and peak locations shifted toward smaller masses by a factor of a few. Assuming that star-forming clumps follow Larson-type relations, we obtain core mass functions in good agreement with the observationally derived IMF, in particular, when taking into account the thermodynamics of the gas. The time-dependent theory directly yields an analytical expression for the star formation rate (SFR) at cloud scales. The SFR values agree well with the observational determinations of various Galactic molecular clouds. Furthermore, we show that the SFR does not simply depend linearly on density, as is sometimes claimed in the literature, but also depends strongly on the clump mass/size, which yields the observed scatter. We stress, however, that any SFR theory depends, explicitly or implicitly, on very uncertain assumptions like clump boundaries or the mass of the most massive stars that can form in a given clump, making the final determinations uncertain by a factor of a few. Finally, we derive a fully time dependent model for the IMF by considering a clump, or a distribution of clumps accreting at a constant rate and thus whose physical properties evolve with time. In spite of its simplicity, this model reproduces reasonably well various features observed in numerical simulations of converging flows. Based on this general theory, we present a paradigm for star formation and the IMF.
\end{abstract}

Key words: ISM: clouds - stars: formation - stars: luminosity function, mass function - turbulence

\section{INTRODUCTION}

Understanding the origin of the initial mass function (IMF) and inferring the star formation rate (SFR) in galaxies are the two main challenges of star formation theory. Many attempts have been made over the years to try to resolve these two fundamental issues (see Hennebelle \& Chabrier 2011a for a brief review regarding the main modern theories of the IMF). Within the last five years, we have developed a new analytical theory, based on the gravo-turbulent paradigm of star formation (e.g., MacLow \& Klessen 2004), aimed at explaining the IMF (Hennebelle \& Chabrier 2008, 2009, hereafter Papers I and II). Indeed, in our theory of the IMF, as well as that of Padoan et al. (1997), largescale supersonic turbulence is supposed to generate small-scale overdense regions with respect to the surrounding background, within which gravity eventually dominates all supports and triggers the collapse, leading to the formation of the prestellar cores. As in Padoan \& Nordlund's theory, we assume that there is a direct correspondence between the prestellar core mass function (CMF) and the final stellar IMF. More precisely, we assume that there is a good correspondence between the mass reservoir out of which the cores form and the IMF; a fact that seems to be supported observationally by the remarkable similarity between the IMF and the CMF (see, e.g., André et al. 2010 for the most recent results concerning this issue), but also statistically by the rather strong correlation between the CMF and the IMF inferred from the analysis of numerical simulations aimed at exploring this issue (Chabrier \& Hennebelle 2010). Recently, Hopkins (2012a, 2012b) derived an IMF theory in

\footnotetext{
5 Visiting scientist, Max Planck Institute for Astrophysics, Garching, Germany.
}

a similar spirit as that of Hennebelle-Chabrier (HC), based on a different, the so-called excursion set formalism, and has extended the results to larger scales, typical of large-scale structures in galactic disks. His results are found to agree fairly well with the $\mathrm{HC}$ ones when considered at the star-forming clump scale.

The theory developed in Papers I and II, which extends to the context of star formation, characterized by nonlinear density fluctuations, the formalism developed in cosmology for linear fluctuations by Press \& Schechter (1974), consists in properly counting at all scales the self-gravitationally bound density fluctuations. ${ }^{6}$ In the $\mathrm{HC}$ theory, these collapsing density fluctuations represent the overdense regions that isolate themselves from the surrounding medium and start to contract under the action of gravity at the very initial stages of star formation, and out of which prestellar cores, and later on individual stars (or multiple star systems such as binaries), will form (see Chabrier \& Hennebelle 2011 for a simplified explanation of the HC formalism). As mentioned in Papers I and II, a limitation so far of this theory resides in the time-independent nature of its formulation. The importance of time dependence for the IMF has been stressed, for instance, by Clark et al. (2007) who argue that since massive (low-density) cores are expected to collapse in a time longer than low-mass (high-density) ones, the CMF should be flatter than the IMF if the latter one is to be inherited from the former one. In the HC theory, however, turbulence-induced velocity dispersion, rather than purely thermal motions, plays a dominant role in setting up the mass of the massive cores when

\footnotetext{
6 As discussed in Sections 3 and 5 of Paper I, the HC theory takes into account the probability for overdense, collapsing structures to be included in larger collapsing ones, and thus properly addresses the so-called cloud in cloud problem present in the Press \& Schechter formalism.
} 
they form, so that the turbulent Jeans mass, which entails a Mach dependence, instead of the thermal Jeans mass, should be used as the characteristic mass scale. This does not mean that turbulence is acting as a pressure or even a support in a static sense, as is often misunderstood, but rather that on large scales, turbulent motions act to prevent larger amounts of material from immediate gravitational collapse. Eventually, for instance where the flows collide, the density is temporarily enhanced, yielding the subsequent collapse of these large mass reservoirs, progenitors of massive cores (see Chabrier \& Hennebelle 2011). Under these conditions, as shown in Appendix C of Paper II, the characteristic free-fall timescale depends much more weakly on the star mass $\left(\tau_{\mathrm{ff}} \propto M^{1 / 4}\right)$, making the aforementioned time problem much less severe. In the present paper, we develop this argument quantitatively by deriving a time-dependent theory of the IMF. We show that, indeed, time dependence barely affects the slope of the IMF at large masses. The peak of the mass spectrum, however, is shifted by a factor of $\simeq 3$ toward smaller masses, as more low-mass prestellar cores are able to form. This timedependent formulation of star formation enables us to derive an SFR. We stress, however, that this SFR is valid at the scale of the clouds and not necessarily at the scale of the entire Galaxy (see, e.g., Ostriker et al. 2010; Renaud et al. 2012).

A summary of the present results and comparisons with previously published SFR theories and with some observational results has been presented in Hennebelle \& Chabrier (2011b, HC11). The present paper presents in detail the whole derivation of our time-dependent IMF theory and of the analytical SFR and confronts the results with further recent observational determinations. The paper is organized as follows. The extension of our formalism to a time-dependent derivation and comparisons between the time-dependent and time-independent theories of the mass spectrum of self-gravitating fluctuations are presented in detail in Section 2, both for the isothermal and non-isothermal cases. The effect of the magnetic field is also considered in this section. In Section 3, we derive the SFR and star formation efficiency. We first derive the theoretical expression and then compute the SFR values for an ensemble of clump parameters and explore the dependence of the SFR upon various clump characteristic properties. ${ }^{7}$ In Section 4 , we make the comparison with recent SFR determinations of molecular clouds in the Galaxy. We briefly discuss the impact of filaments in star formation upon our formalism. In Section 5, we present the complete self-consistent time-dependent model by exploring the consequences of our time-dependent theory on the mass spectrum of different evolving clumps. Then, we investigate the impact on the mass spectrum of a time-dependent clump distribution instead of a single clump. Section 6 is devoted to the conclusion and our paradigm for star formation and the IMF is presented.

\section{TIME-DEPENDENT THEORY}

\subsection{Analytical Expression of the Mass Spectrum}

The underlying concept on which the theory developed in Papers I and II relies consists of identifying, in a random field of density fluctuations, the mass $M_{R}$ which at scale $R$ (physically speaking, $R$ denotes the radius of the density fluctuation) is gravitationally unstable, i.e., the mass contained in regions within which gravity dominates over all sources of support.

\footnotetext{
7 In our theory (see Papers I and II), star-forming "clumps" are identified as overdense $\left(\bar{n} \geq 10^{2}-10^{3} \mathrm{~cm}^{-3}\right) \sim 1-10 \mathrm{pc}$ size unbound regions within large, diffuse molecular clouds within which $\sim 0.1 \mathrm{pc}$ size gravitationally bound prestellar "cores" will preferentially form.
}

To achieve this, the first step is to determine $M_{R}$, the mass contained in regions whose density exceeds a scale-dependent density threshold, $\log \delta_{R}^{c}$, determined by the virial condition. Since all this gas is unstable at a scale smaller than or equal to $R$, it is expected that it will end up in objects of mass smaller than or equal to the Jeans mass associated with the density threshold. Thus, the second step is to make $M_{R}$ equal with the mass contained in the structures of mass equal to or smaller than the associated (turbulent) Jeans mass (see Equations (28) and (29) of Paper I).

This approach implicitly assumes that each part of the flow is initially assigned a specific (scale-dependent) Jeans mass, which collapses. While such an assumption is certainly reasonable in the case of cold dark matter fluctuations within the primordial universe, it is a priori not the case in a turbulent flow in which fluctuations of scale $R$ are replenished within a few crossing times, $\tau_{R} \simeq 2 R / V_{\text {rms }}(R)$, where $V_{\text {rms }}(R) \equiv\left\langle\sigma_{R}^{2}\right\rangle^{1 / 2}$ denotes the turbulent rms velocity at scale $R$ and $\sigma_{R}$ the velocity fluctuation over scales smaller than $R$. Therefore, in the case of turbulent molecular clumps, it seems necessary to take such dynamical effects into account. This is not obvious by any means, in particular for large-scale fluctuations for which, first, a long enough time is required and, second, gravity must not entirely freeze the gas motions by preventing fluid particles to escape their local Jeans masses. In this latter case, gravity will prevent the flow motions and the replenishment of the density fluctuations, which have become gravitationally unstable.

On the other hand, if the clump is accreting at a sufficiently high rate or if it contains a large fraction of gas that is not dense enough to be locally gravitationally unstable (i.e., if the Jeans length at the gas density is comparable to or larger than the size of the clump), then dissipation of large-scale turbulence leads to turbulent compression which can continuously generate density fluctuations at all scales and trigger, for the densest ones, gravitational collapse. In this case, one must take into account the fact that during the lifetime of the cloud, $\tau_{0}$, the fluctuations at scale $R$ have been replenished a number of times equal to $\tau_{0} / \tau_{R}$. This implies that Equation (31) of Paper I must be modified as

$$
\begin{aligned}
\frac{M_{\mathrm{tot}}(R)}{V_{c}} & =\int_{\delta_{R}^{c}}^{\infty} \bar{\rho} \exp (\delta) \mathcal{P}_{R}(\delta)\left(\frac{\tau_{0}}{\tau_{R}}\right) d \delta \\
& =\int_{0}^{M_{R}^{c}} M^{\prime} \mathcal{N}\left(M^{\prime}\right) P\left(R, M^{\prime}\right) d M^{\prime},
\end{aligned}
$$

where $\delta=\log (\rho / \bar{\rho})$ denotes the (logarithm of) density fluctuation, $\delta_{R}^{c}$ is the threshold density at scale $R, \mathcal{P}(\delta)$ is the density distribution (PDF), ${ }^{8}$ and $V_{c} \sim L_{c}^{3}$ denotes the clump's volume.

Apart from the term $\tau_{0} / \tau_{R}$, this equation is the same as that derived in Paper I. It uses the formulation developed by Jedamzik (1995) in the context of dark matter halos (see also Yano et al. 1996). The first equality for $M_{\text {tot }}(R)$ stems from the fact that the mass contained within structures of mass $M<M_{R}^{c}$ is equal to the mass of the gas which, smoothed at scale $R$, has a (logarithmic) density larger than a critical threshold $\delta_{R}^{c}$. The second expression arises from the fact that the number density of structures of mass $M<M_{R}^{c}$ is $\mathcal{N}\left(M^{\prime}\right) P\left(R, M^{\prime}\right) d M^{\prime}$. Here, $\mathcal{N}\left(M^{\prime}\right) d M^{\prime}$ is the number density of structures of mass between $M^{\prime}$ and $M^{\prime}+d M^{\prime}$, while $P\left(R, M^{\prime}\right)$ is the probability of finding a gravitationally unstable structure of mass $M^{\prime}$ embedded inside a structure of gas which at scale $R$ has a (logarithmic) density

\footnotetext{
8 We stress that, in our general formalism, $\mathcal{P}$ is not necessarily a lognormal, as emphasized in Schmidt et al. (2010).
} 
larger than $\delta_{R}^{c} . P\left(R, M^{\prime}\right)$ is assumed to be equal to 1 (see Appendix D of Paper I for further justification). The time ratio $\tau_{0} / \tau_{R}$ thus simply illustrates the fact that the flow fluctuations at scale $R$ have been rejuvenated $\tau_{0} / \tau_{R}$ times.

Taking the derivative of Equation (1) with respect to $R$, we obtain for the number-density mass spectrum of gravitationally bound structures, $\mathcal{N}(M)=d\left(N / V_{c}\right) / d M$ :

$$
\begin{aligned}
\mathcal{N}\left(M_{R}^{c}\right)= & \frac{\bar{\rho}}{M_{R}^{c}} \frac{d R}{d M_{R}^{c}}\left(-\frac{d \delta_{R}^{c}}{d R}\left(\frac{\tau_{0}}{\tau_{R}}\right) \exp \left(\delta_{R}^{c}\right) \mathcal{P}_{R}\left(\delta_{R}^{c}\right)\right. \\
& \left.+\int_{\delta_{R}^{c}}^{\infty} \exp (\delta) \frac{d}{d R}\left[\left(\frac{\tau_{0}}{\tau_{R}}\right) \mathcal{P}_{R}\right] d \delta\right)
\end{aligned}
$$

As shown in Paper I, the first term in this expression is the most important one and dominates over the second one except when the scale $R$ becomes comparable to the injection scale, $L_{i}$, which is basically the size of the cloud (see the interesting formulation of Hopkins 2012a, 2012b to avoid this large-scale limitation of the present formalism).

Once the expression of the critical density threshold, $\delta_{R}^{c}=$ $\ln (\rho(R) / \bar{\rho})$, is specified from the virial condition, $\left\langle V_{\mathrm{rms}}^{2}(R)\right\rangle+$ $3 C_{S}^{2}<E_{\mathrm{pot}}(R) / M$ (see Section 4.2 of Paper I), the mass spectrum of the self-gravitating pieces of fluid can be inferred from

$$
M_{R}=C_{m} \rho(R) R^{3}
$$

where $C_{m}$ is a geometrical factor, typically of the order of $4 \pi / 3$.

Before proceeding further, it is worth stressing what is exactly selected by our procedure. As seen from Equation (1), the integration is performed from 0 to $M_{R}^{c}$. This means that the smallest Jeans masses, corresponding to the densest pieces of gas, are first accounted for and removed from the available gas mass. Then the larger Jeans masses are progressively taken into account and removed. By doing so, we properly take into account the fact that there are small Jeans masses embedded into larger ones. This happens, in particular, when the PDF significantly varies locally, i.e., when turbulence is strong, which implies that in that case the scale must be large. Therefore, strictly speaking, we do not identify well-defined bound cores but rather coherent mass reservoirs in the density field that isolate themselves from the surrounding medium under the action of gravity. For sufficiently small scales, the density becomes reasonably uniform within the mass reservoir to have a clear correspondence between this latter and a wellidentified "core." For large scales, which will lead eventually to the formation of massive stars, however, the one-to-one core-reservoir correspondence becomes more blurry (as the potential well associated with the mass reservoir becomes itself more shallow). In that case, the reservoir of mass out of which the most massive cores/stars will form corresponds to what is left once all the self-gravitating small-scale density fluctuations embedded in the reservoir have been properly accounted for and "taken away." In that sense, for the largest scales, and thus the most massive cores, our formalism rejoins in some sense the so-called competitive accretion process (see, e.g., Smith et al. 2009). Therefore, in principle, the turbulent fragmentation process is properly described in our formalism, apart from the fact that for the sake of simplicity, the conditional probability $P(R, M)$ is taken to be equal to 1 . What has not been taken into account so far, however, is the gravitational fragmentation that occurs during the collapse. Although such fragmentation may occur, its importance is likely to remain limited because of the impending roles of the magnetic field (Machida et al. 2005, 2008; Hennebelle \& Teyssier 2008; Price \& Bate 2008; Hennebelle et al. 2011) and radiation (Krumholz et al. 2007; Bate 2009; Commercon et al. 2010), particularly when both are present (Commercon et al. 2011), an issue which still needs to be properly quantified. Observations of massive cores indeed suggest that fragmentation is rather limited, with most of the mass of the core ending up in one or just a few smaller cores (Bontemps et al. 2010; Longmore et al. 2011; Bressert et al. 2010; Palau et al. 2013).

\subsection{Influence of the Time Dependence on the $C M F / I M F$ : Isothermal Case}

In this section, we examine the impact of time dependence on the CMF in the simple isothermal case. The barotropic case will be examined in Section 2.3. We first discuss the density PDF, then the crossing time. Finally, we derive the analytical expression and compare the results with the time-independent ones.

\subsubsection{Probability Density Function}

Numerical simulations (Vázquez-Semadeni 1994; Padoan et al. 1997; Passot \& Vázquez-Semadeni 1998; Kritsuk et al. 2007; Federrath et al. 2008; Schmidt et al. 2009) have revealed that the turbulence-induced density distribution, $\mathcal{P}(\delta)$, is reasonably well described by a lognormal distribution

$$
\begin{aligned}
\mathcal{P}(\delta) & =\frac{1}{\sqrt{2 \pi \sigma_{0}^{2}}} \exp \left(-\frac{(\delta-\bar{\delta})^{2}}{2 \sigma_{0}^{2}}\right), \\
\delta & =\ln (\rho / \bar{\rho}), \quad \bar{\delta}=-\sigma_{0}^{2} / 2, \quad \sigma_{0}^{2}=\ln \left(1+b^{2} \mathcal{M}^{2}\right) .
\end{aligned}
$$

In this expression, $\mathcal{M}$ is the characteristic cloud Mach number and $b$ is a non-dimensional coefficient that depends on the turbulence forcing (see Federrath et al. 2010). It typically varies from 0.25 when the forcing is purely solenoidal to almost 1 when the forcing is applied only on compressible modes. Such a three-dimensional (3D) lognormal shape for density fluctuations has received observational support from its two-dimensional (2D) observed projection, namely, the power spectrum column density of molecular clouds, measured from dust extinction maps (Kainulainen et al. 2009; Brunt et al. 2010). The scale dependence of the variance of the distribution reads, in 3D (see Paper I),

$$
\sigma^{2}(R)=\int_{2 \pi / L_{c}}^{2 \pi / R} \tilde{\delta}(k)^{2} 4 \pi k^{2} d k=\sigma_{0}^{2}\left(1-\left(\frac{R}{L_{c}}\right)^{n^{\prime}-3}\right),
$$

where $L_{c}$ is the cloud's size and $\widetilde{\delta}(k)^{2} \propto k^{-n^{\prime}}$ is the power spectrum of $\log (\rho)$ of 3D index $n^{\prime}$. This latter is found in isothermal, shock-dominated hydrodynamical and MHD simulations to be very similar to the index $n$ of the velocity power spectrum, with a typical value $n^{\prime} \sim n \sim 3.8$, between 11/3 (Kolmogoroff limit) and 4 (Burgers limit; see, e.g., Kritsuk et al. 2007; Federrath et al. 2010; Schmidt et al. 2009), although the exact value depends on the forcing. Then (see Paper I),

$$
\mathcal{P}(\delta)=\frac{1}{\sqrt{2 \pi \sigma(R)^{2}}} \exp \left(-\frac{\left[\delta+\frac{\sigma(R)^{2}}{2}\right]^{2}}{2 \sigma(R)^{2}}\right) .
$$




\subsubsection{The Crossing Time, $\tau_{R}$}

The crossing time at scale $R$ is given by $\tau_{R} \equiv \tau_{\mathrm{ct}}(R)=$ $2 \alpha_{\mathrm{ct}} R / V_{\mathrm{ct}}$, where $V_{\mathrm{ct}}$ is the relevant velocity and $\alpha_{\mathrm{ct}}$ a dimensionless coefficient of the order of a few. At large scales, $V_{\text {ct }}$ is typically equal to the $1 \mathrm{D}$ velocity dispersion $V_{\mathrm{rms}}^{1 \mathrm{D}}=V_{\mathrm{rms}} / \sqrt{3}$, where $V_{\text {rms }} \equiv V_{\text {rms }}^{3 \mathrm{D}}$ designates the $3 \mathrm{D}$ velocity dispersion all along this paper, while at small scales, below the sonic length, $V_{\text {ct }} \simeq C_{s}$. This crossing time is the typical time that is necessary for the density field to be significantly modified at scale $R$, implying that a new set of fluctuations, statistically independent of the former one, has been processed.

As mentioned earlier and discussed in Papers I and II, in the present context, we select the pieces of gas which are selfgravitating, i.e., such that their internal gravitational, kinetic, and thermal energies obey the following condition: $-E_{\text {grav }}>$ $2 E_{\text {kin }}+3 P_{\text {th }}$. At large scales, this implies that $\alpha_{g} G M / R>V_{\text {rms }}^{2}$, where $\alpha_{g}$ is a dimensionless coefficient equal to $3 / 5$ for a uniform density fluctuation. We thus get

$$
\tau_{R}=\frac{2 \alpha_{\mathrm{ct}} R}{V_{\mathrm{rms}}^{1 \mathrm{D}}}=2 \alpha_{\mathrm{ct}} \sqrt{\frac{24}{\pi^{2} \alpha_{g}}} \tau_{\mathrm{ff}}(R),
$$

where $\tau_{\mathrm{ff}}(R)=\sqrt{3 \pi / 32 G \rho(R)}$ is the free-fall time of the density fluctuation of density $\rho(R)$. A similar expression is obtained below the sonic length. In the following, we thus define the crossing time of a collapsing density fluctuation of scale $R$ as

$$
\tau_{R}=\phi_{t} \tau_{\mathrm{ff}}(R)
$$

$$
\text { with } \phi_{t}=2 \alpha_{\mathrm{ct}} \sqrt{\frac{24}{\pi^{2} \alpha_{g}}} \simeq 3 \text {. }
$$

Note that Federrath $\&$ Klessen (2012) estimated $\phi_{t} \simeq 2$ from a fit between simulations and observations.

Defining $\tau_{\mathrm{ff}}^{0}$ as the free-fall time at the clump's mean density, $\tau_{\mathrm{ff}}^{0}=\sqrt{3 \pi / 32 G \bar{\rho}} \simeq 1.07(\mu / 2.33)^{-1 / 2}\left(\bar{n} / 10^{3} \mathrm{~cm}^{-3}\right)^{-1 / 2} \mathrm{Myr}$ (where $\mu=2.33$ is the mean molecular weight for a cosmic $\mathrm{H}_{2}$ /He composition), we get

$$
\frac{\tau_{R}}{\tau_{\mathrm{ff}}^{0}}=\phi_{t} \sqrt{\frac{\bar{\rho}}{\rho}} .
$$

Note that the choice of $\tau_{\mathrm{ff}}^{0}$ is not consequential at this stage as it simply modifies the value of $\mathcal{N}$ uniformly without affecting its shape. Combining Equations (2), (6), and (9) and dropping the second term in Equation (2), as mentioned earlier, and assuming that $\tau_{\mathrm{ff}}^{0} \simeq \tau_{0}$, we obtain (see Paper I)

$$
\begin{aligned}
\mathcal{N}(\tilde{M})= & -\frac{1}{\phi_{t}} \frac{\bar{\rho}}{M_{J}^{0} \widetilde{M}}\left(\frac{\widetilde{M}_{R}^{c}}{\widetilde{R}^{3}}\right)^{1 / 2} \frac{d \widetilde{R}}{d \widetilde{M}_{R}^{c}} \frac{d \delta_{R}^{c}}{d \widetilde{R}} \frac{1}{\sqrt{2 \pi \sigma^{2}}} \\
& \times \exp \left(-\frac{\left(\delta_{R}^{c}\right)^{2}}{2 \sigma^{2}}+\frac{\delta_{R}^{c}}{2}-\frac{\sigma^{2}}{8}\right),
\end{aligned}
$$

where $M_{R}^{c}$ is the critical mass at scale $R$ (see Equation (3)) while

$$
\delta_{R}^{c}=\ln \left(\frac{\widetilde{M}_{R}^{c}}{\widetilde{R}^{3}}\right),
$$

where $\widetilde{R}=R / \lambda_{J}^{0}, \widetilde{M}=M / M_{J}^{0}$, and $M_{J}^{0}$ and $\lambda_{J}^{0}$ denote the usual mean thermal Jeans mass and Jeans length, respectively:

$$
\begin{aligned}
M_{J}^{0} & =\frac{a_{J}}{C_{m}} \frac{C_{s}^{3}}{\sqrt{G^{3} \bar{\rho}}} \\
& \approx 0.8\left(\frac{a_{J}}{C_{m}}\right)\left(\frac{T}{10 \mathrm{~K}}\right)^{3 / 2}\left(\frac{\mu}{2.33}\right)^{-2}\left(\frac{\bar{n}}{10^{4} \mathrm{~cm}^{-3}}\right)^{-1 / 2} M_{\odot}
\end{aligned}
$$

$$
\begin{aligned}
\lambda_{J}^{0} & =\left(\frac{\pi^{3 / 2}}{C_{m}}\right)^{1 / 3} \frac{C_{s}}{\sqrt{G \bar{\rho}}} \\
& \approx 0.2\left(\frac{\pi^{3 / 2}}{C_{m}}\right)^{1 / 3}\left(\frac{T}{10 \mathrm{~K}}\right)^{1 / 2}\left(\frac{\mu}{2.33}\right)^{-1}\left(\frac{\bar{n}}{10^{4} \mathrm{~cm}^{-3}}\right)^{-1 / 2} \mathrm{pc},
\end{aligned}
$$

where $a_{J}$ is a dimensionless geometrical factor of the order of unity. Taking the standard definition of the Jeans mass as the mass enclosed in a sphere of diameter equal to the Jeans length, one gets $a_{J}=\pi^{5 / 2} / 6$. We now need to know $M_{R}^{c}$, and its derivative $d M_{R}^{c} / d R$, as a function of the scale, $R$. It is determined by the physical processes at play in the cloud, as examined in the next sections.

\subsubsection{Analytical Expression}

We now need to specify the density threshold, $\delta_{R}^{c}$, which is determined from the virial theorem. As shown in Paper I (Section 4.3), the condition for collapse, which simply reads $\delta>\delta_{R}^{c}$ or equivalently $M>M_{R}^{c}=M_{J}(R)$, yields after calculations

$$
M>a_{J} \frac{\left[\left(C_{s}(\rho)\right)^{2}+\left(\frac{V_{\mathrm{rms}}^{2}}{3}\right)\right]^{3 / 2}}{\sqrt{G^{3} \bar{\rho} \exp (\delta)}} .
$$

We assume that the effective dispersion velocity obeys the Larson's (1981) relationship:

$$
\begin{aligned}
\left\langle V_{\mathrm{rms}}^{2}\right\rangle & =V_{0}^{2} \times\left(\frac{R}{1 \mathrm{pc}}\right)^{2 \eta}, \\
\mathcal{M} & \equiv \mathcal{M}(R)=\frac{\left\langle V_{\mathrm{rms}}^{2}\right\rangle^{1 / 2}}{C_{s}} .
\end{aligned}
$$

As mentioned in Paper I, the Mach number $\mathcal{M}$ represents "effective" values that include both the hydrodynamical and magnetic contributions, i.e., $V_{\mathrm{rms}}=\left\{\left(V_{\mathrm{rms}}\right)_{\text {hydro }}^{2}+V_{A}^{2} / 2\right\}^{1 / 2}$, where $V_{A}=B /(4 \pi \rho)^{1 / 2}$ denotes the Alfvén velocity. The coefficient $\eta$ is related to $n$, the index of the velocity power spectrum, by the relation (see Equation (24) of Paper I)

$$
\eta=\frac{n-3}{2} .
$$

As mentioned earlier, 3D numerical simulations of compressible turbulence (e.g., Kritsuk et al. 2007) suggest a value $n \simeq 3.8$, yielding $\eta \simeq 0.4$, as is indeed found in observations.

With Equation (3), Equation (15) implies (see Paper I)

$$
M>M_{R}^{c}=a_{J}^{2 / 3}\left(\frac{C_{s}^{2}}{G} R+\frac{V_{\mathrm{rms}}^{2}}{3 G}\right) .
$$


After normalization, Equation (18) becomes (see Section 5.4 of Paper I)

$$
\widetilde{M}_{R}^{c}=M / M_{J}^{0}=\widetilde{R}\left(1+\mathcal{M}_{*}^{2} \widetilde{R}^{2 \eta}\right),
$$

where $\mathcal{M}_{*}$ is given by

$$
\begin{aligned}
\mathcal{M}_{*} & =\frac{1}{\sqrt{3}} \frac{V_{0}}{C_{s}}\left(\frac{\lambda_{J}^{0}}{1 \mathrm{pc}}\right)^{\eta} \\
& \simeq(0.8-1.0)\left(\frac{\lambda_{J}^{0}}{0.1 \mathrm{pc}}\right)^{\eta}\left(\frac{C_{s}}{0.2 \mathrm{~km} \mathrm{~s}^{-1}}\right)^{-1},
\end{aligned}
$$

and thus illustrates the impact of turbulence-induced velocity fluctuations at the Jeans scale (see Paper I).

Equations (11) and (19) finally yield for the mass spectrum of gravitationally bound prestellar cores:

$$
\begin{aligned}
\mathcal{N}(\tilde{M})= & \frac{2}{\phi_{t}} \mathcal{N}_{0} \frac{1}{\widetilde{R}^{6}} \frac{1+(1-\eta) \mathcal{M}_{*}^{2} \widetilde{R}^{2 \eta}}{\left[1+(2 \eta+1) \mathcal{M}_{*}^{2} \widetilde{R}^{2 \eta}\right]} \\
& \times\left(\frac{\widetilde{M}}{\widetilde{R}^{3}}\right)^{-1-\frac{1}{2 \sigma^{2}} \ln \left(\widetilde{M} / \widetilde{R}^{3}\right)} \times \frac{\exp \left(-\sigma^{2} / 8\right)}{\sqrt{2 \pi} \sigma},
\end{aligned}
$$

where $\mathcal{N}_{0}=\bar{\rho} / M_{J}^{0}$. Equation (21) is the time-dependent generalization of Equation (44) of Paper I. The time dependence appears explicitly through the factor $1 / \phi_{t}$ but also through the modification of the exponent, $-1-(1 / 2) \sigma^{2} \ln \left(\widetilde{M} / \widetilde{R}^{3}\right)$, instead of $-3 / 2-(1 / 2) \sigma^{2} \ln \left(\widetilde{M} / \widetilde{R}^{3}\right)$, which arises from the time correction, proportional to $\sqrt{\rho} \propto\left(M / R^{3}\right)^{1 / 2}$. As expected from the discussion in the Introduction, the time dependence thus affects the slope of the CMF. We quantify this effect in the next sections.

\subsubsection{Results and Comparison with the Time-independent Model}

Before comparing the time-dependent and time-independent distributions, we determine the position of the peak of the CMF. As discussed in Paper I (Section 7.1.4), the peak occurs in the thermally dominated regime, which corresponds to $\mathcal{M}_{*}=0$. The derivative of Equation (21) with respect to the mass in that case yields

$$
\tilde{M}_{\text {peak }}=\frac{M_{\text {peak }}}{M_{J}^{0}}=\exp \left(-\sigma^{2}\right)=\frac{1}{\left(1+b^{2} \mathcal{M}^{2}\right)},
$$

whereas in the case of a time-independent distribution we have (Equation (46) Paper I)

$$
\widetilde{M}_{\text {peak }}=\exp \left(-\frac{3}{4} \sigma^{2}\right)=\frac{1}{\left(1+b^{2} \mathcal{M}^{2}\right)^{3 / 4}}
$$

This implies that for a given Mach number, the peak of the distribution is shifted toward smaller masses when time dependence is taken into account. This is intuitively expected from the fact that more small-scale objects with short free-fall times are continuously produced during the clump evolution in a time-dependent collapse. It is thus important to stress that in the (both time-dependent and time-independent) HC theory of gravo-turbulent fragmentation, the peak (i.e., the characteristic) mass of the IMF depends not only on the clump's mean thermal Jeans mass but also strongly $\left(\propto \mathcal{M}^{-2}\right)$ on the characteristic Mach number.

Another important quantity is the exponent of the high-mass tail of the distribution. Indeed, in the limit $\widetilde{M} \simeq \mathcal{M}_{*}^{2} \widetilde{R}^{2 \eta+1}$,
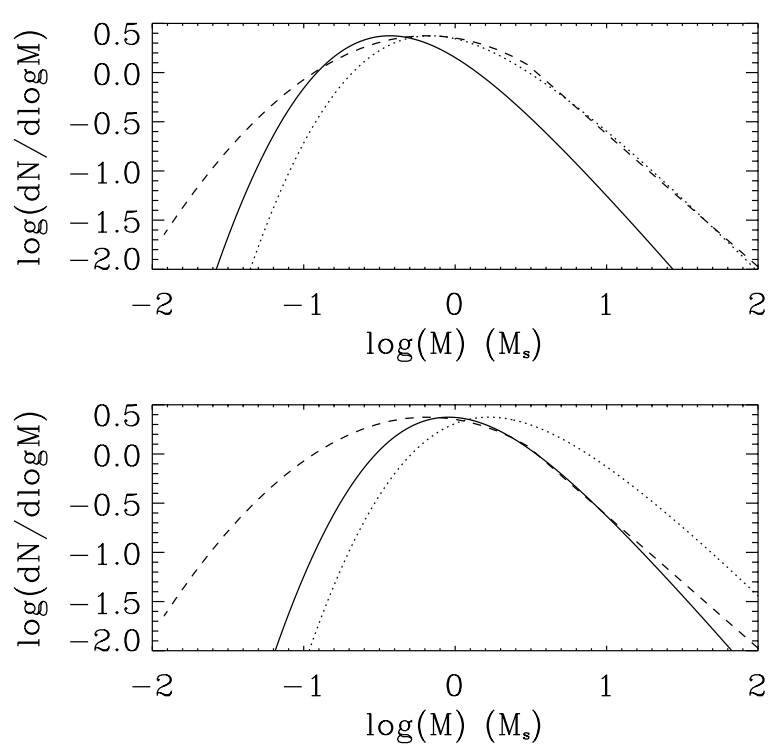

Figure 1. Isothermal case. Core mass function, $d N / d \log M$, for $\mathcal{M}=6$, $\mathcal{M}_{*}=\sqrt{2}$, and mean clump density $\bar{n}=3 \times 10^{4} \mathrm{~cm}^{-3}$ (top panel) and $\bar{n}=5 \times 10^{3} \mathrm{~cm}^{-3}$ (bottom panel). The solid line corresponds to the timedependent model while the dotted line represents the time-independent one. The dashed line is the Chabrier IMF shifted upward in mass by a factor of three (see the text).

which corresponds to the turbulence-dominated regime (see Equation (19)), Equation (21) tends to a power law plus a lognormal, such as $\mathcal{N} \propto M^{-(1+x)}$ with

$$
x=\frac{3}{2 \eta+1}-6 \frac{1-\eta}{(2 \eta+1)^{2} \sigma^{2}} \ln \left(\mathcal{M}_{*}\right),
$$

whereas in the time-independent case, this coefficient reads (see Equation (43) of Paper II)

$$
x=\frac{2+\eta}{2 \eta+1}-6 \frac{1-\eta}{(2 \eta+1)^{2} \sigma^{2}} \ln \left(\mathcal{M}_{*}\right) .
$$

As expected, the distribution becomes slightly steeper when the time dependence is included in the derivation of the CMF/IMF. This is due to the fact that some massive clumps fragment into smaller pieces during the cloud collapse. Typical values for $x$ range from 1.1 to 1.5 , depending on $\eta, \sigma$, and $\mathcal{M}_{*}$, bracketing the Salpeter value, $x=1.35$.

Figure 1 displays the core mass function, $d N / d \log M$ for $\eta=0.45, \mathcal{M}=6, \mathcal{M}_{*}=\sqrt{2}$, and two typical clump densities, $\bar{n}=5000 \mathrm{~cm}^{-3}$ and $\bar{n}=3 \times 10^{4} \mathrm{~cm}^{-3}$. Solid lines display the time-dependent results, while dotted lines display the timeindependent ones. For reference, dashed lines represent the Chabrier system IMF (Chabrier 2003), shifted upward in mass along the $x$-axis by a factor of three to account for the observed shift between the CMF and the IMF. In the rest of the paper, we will refer to this IMF as the "shifted Chabrier IMF" (SCIMF). As mentioned above, the time-dependent distribution peaks at lower masses and has a slightly steeper high-mass slope than the time-independent one. In spite of these differences, both distributions match well the SCIMF above about the mean Jeans mass. At low masses, both distributions appear to be too narrow. As already discussed in Papers I and II, this is essentially due to the isothermal approximation for the equation of state (eos) of the gas (see Paper II and below), but also to the assumed exact correspondence between the initial core mass and the final star mass. Indeed, as shown in Chabrier \& Hennebelle (2010), taking 
into account some (expected) dispersion between the mass of the parent core and that of the final star, i.e., between the CMF and the IMF, naturally leads to a broadening of the latter compared with the former in the low-mass regime. At last, gravity may also broaden the PDF.

\subsection{Barotropic Equation of State and Magnetic Field}

Here we compare the time-dependent and time-independent models when a barotropic eos is used. We also introduce the magnetic field in our model and investigate its impact on the mass spectrum.

\subsubsection{Formalism}

Again, assuming that the pieces of fluid that collapse are gravitationally dominated, Equation (15) becomes

$$
M>a_{J} \frac{\left[\left(C_{s}(\rho)\right)^{2}+\left(V_{\mathrm{rms}}^{2} / 3\right)+\left(V_{a}^{2} / 6\right)\right]^{3 / 2}}{\sqrt{G^{3} \bar{\rho} \exp (\delta)}},
$$

where $V_{a}$ is the mean Alfvén speed. Based on observational and numerical results, we assume that the magnetic intensity correlates with the gas density as

$$
B=B_{0}\left(\frac{\rho}{\bar{\rho}}\right)^{\gamma_{b}}
$$

Typical values for $B_{0}$ and $\bar{\rho}$ are $10-20 \mu \mathrm{G}$ and $10^{3} \mathrm{~cm}^{-3}$, respectively (Crutcher 1999). The coefficient $\gamma_{b}$ seems to depend on density. In the diffuse gas, $\gamma_{b}$ is typically smaller than 0.5 and around 0.1-0.3 (Troland \& Heiles 1986; Hennebelle et al. 2008), while for higher densities when the gas is self-gravitating, it is of the order of 0.5 (although see Crutcher et al. 2010).

After normalization, Equation (26) becomes

$$
\tilde{M}_{R}^{c}=M / M_{J}^{0}=\widetilde{R}\left(\frac{C_{s}(\rho)^{2}}{\left(C_{s}^{0}\right)^{2}}+\mathcal{M}_{*}^{2} \widetilde{R}^{2 \eta}+\left(V_{a}^{*}\right)^{2}\left(\frac{\rho}{\bar{\rho}}\right)^{2 \gamma_{b}-1}\right),
$$

where

$$
V_{a}^{*}=\frac{1}{\sqrt{6}} \frac{B_{0}}{\sqrt{4 \pi \bar{\rho}} C_{s}} .
$$

As emphasized in Paper II (see also Hennebelle 2012 for a self-consistent calculation of the thermal balance), the thermodynamics of the gas has a drastic impact on the low-mass end of the mass function. As in Paper II, we use the barotropic eos suggested by Larson (1985, see also Glover \& Mac Low 2007) from observations of molecular clouds, namely,

$$
\begin{array}{ll}
T \propto \rho^{\gamma_{1}-1} & \rho<\rho^{\text {crit }}, \\
T \propto \rho^{\gamma_{2}-1} & \rho>\rho^{\text {crit }},
\end{array}
$$

with $\gamma_{1} \simeq 0.7$ and $\gamma_{2} \simeq 1-1.1$, and $\rho^{\text {crit }}$ corresponds to the density above which dust grains become thermally coupled with the gas and thus dust cooling becomes the dominant cooling mechanism instead of line cooling. Observations suggest that $\rho^{\text {crit }} \simeq 10^{-18} \mathrm{~g} \mathrm{~cm}^{-3}$, i.e., $\bar{n}^{\text {crit }} \simeq 2.5 \times 10^{5} \mathrm{~cm}^{-3}$. We keep the same eos as we used in Paper I, inspired from Larson (1985) and write

$$
\begin{aligned}
C_{s}^{2} & =\left[\left(\left(C_{s, 1}^{0}\right)^{2}\left(\frac{\rho}{\bar{\rho}}\right)^{\gamma_{1}-1}\right)^{m}+\left(\left(C_{s, 2}^{0}\right)^{2}\left(\frac{\rho}{\bar{\rho}}\right)^{\gamma_{2}-1}\right)^{m}\right]^{1 / m} \\
& =\left(C_{s, 1}^{0}\right)^{2}\left[\left(\frac{\rho}{\bar{\rho}}\right)^{\left(\gamma_{1}-1\right) m}+\left(\frac{C_{s, 2}^{0}}{C_{s, 1}^{0}}\right)^{2 m}\left(\frac{\rho}{\bar{\rho}}\right)^{\left(\gamma_{2}-1\right) m}\right]^{1 / m},
\end{aligned}
$$

where $m$ is a real number of the order of unity. In the following, $m$ will be equal to 3 (see Figure 4 of Paper II). Typically, $\gamma_{1} \simeq 0.7$ while $\gamma_{2} \simeq 1-1.1$. The critical density, $\rho^{\text {crit }}$, at which the transition between the two regimes is occurring is expected to be about $10^{-18} \mathrm{~g} \mathrm{~cm}^{-3}$ and we define

$$
K_{\text {crit }}=\left(\frac{C_{s, 2}^{0}}{C_{s, 1}^{0}}\right)^{2}=\left(\frac{\rho^{\text {crit }}}{\bar{\rho}}\right)^{\gamma_{1}-\gamma_{2}} .
$$

In order to get the mass spectrum from Equation (11), we need to know $M_{R}^{c}$ and $d M_{R}^{c} / d R$ as a function of $R$. While for the first it is not possible to get an explicit relation from Equation (28), the second can be obtained by deriving Equation (28) with respect to $\widetilde{R}$. The analytical expressions that we obtain are identical to Equation (38) of Paper II except for the extra terms related to the magnetic field. We write them explicitly for completeness:

$$
\begin{gathered}
\frac{d \widetilde{M}_{R}^{c}}{d \widetilde{R}}=\frac{B}{C} \\
B=D-3 \frac{\widetilde{M}}{\widetilde{R}^{3}} \frac{d D}{d \widetilde{\rho}}+(2 \eta+1) \mathcal{M}_{*}^{2} \widetilde{R}^{2 \eta} \\
C=1-\widetilde{R}^{-2} \frac{d D}{d \widetilde{\rho}}, \\
D=\left(\widetilde{\rho}^{\left(\gamma_{1}-1\right) m}+\left(K_{\text {crit }}\right)^{m} \widetilde{\rho}^{\left(\gamma_{2}-1\right) m}\right)^{1 / m}+\left(V_{a}^{*}\right)^{2} \widetilde{\rho}^{2 \gamma_{b}-1} . \\
\text { 2.3.2. Comparison between Time-dependent and Time-independent } \\
\text { Mass Spectra in the Barotropic Case }
\end{gathered}
$$

When a barotropic eos is considered, the mass spectrum depends on the clump's mean density not only as a normalization factor (as for the isothermal eos) but also through the eos itself. As a fiducial value, we adopt a critical density (Equation (32)) $\bar{n}^{\text {crit }}=2 \times 10^{5} \mathrm{~cm}^{-3}$, i.e., $\rho^{\text {crit }} \simeq 10^{-18} \mathrm{~g} \mathrm{~cm}^{-3}$, as inferred from observations. The density and velocity dispersions are taken to follow Larson- (1981; see also Falgarone et al. 2004 where slightly different values are inferred) type relations:

$$
\begin{aligned}
\bar{n} & =\left(d_{0} \times 10^{3} \mathrm{~cm}^{-3}\right)\left(\frac{R}{1 \mathrm{pc}}\right)^{-0.7}, \\
V_{\mathrm{rms}} & =\left(u_{0} \times 0.8 \mathrm{~km} \mathrm{~s}^{-1}\right)\left(\frac{R}{1 \mathrm{pc}}\right)^{\eta} .
\end{aligned}
$$

According to Equation (16), $V_{0}=u_{0} \times 0.8 \mathrm{~km} \mathrm{~s}^{-1}$. Figure 2 (left panel) shows the mass spectrum for four clumps of size $R_{c}=0.5,2,5$, and $20 \mathrm{pc}$, respectively. The value of $u_{0}$ is taken to be equal to 1 in all cases, while the top, middle, and bottom panels correspond to $d_{0}=5,4,3$, and 2, respectively. The four panels thus correspond, from top to bottom, to typical clump masses and mean densities, $M_{c}=183$, 3564, 

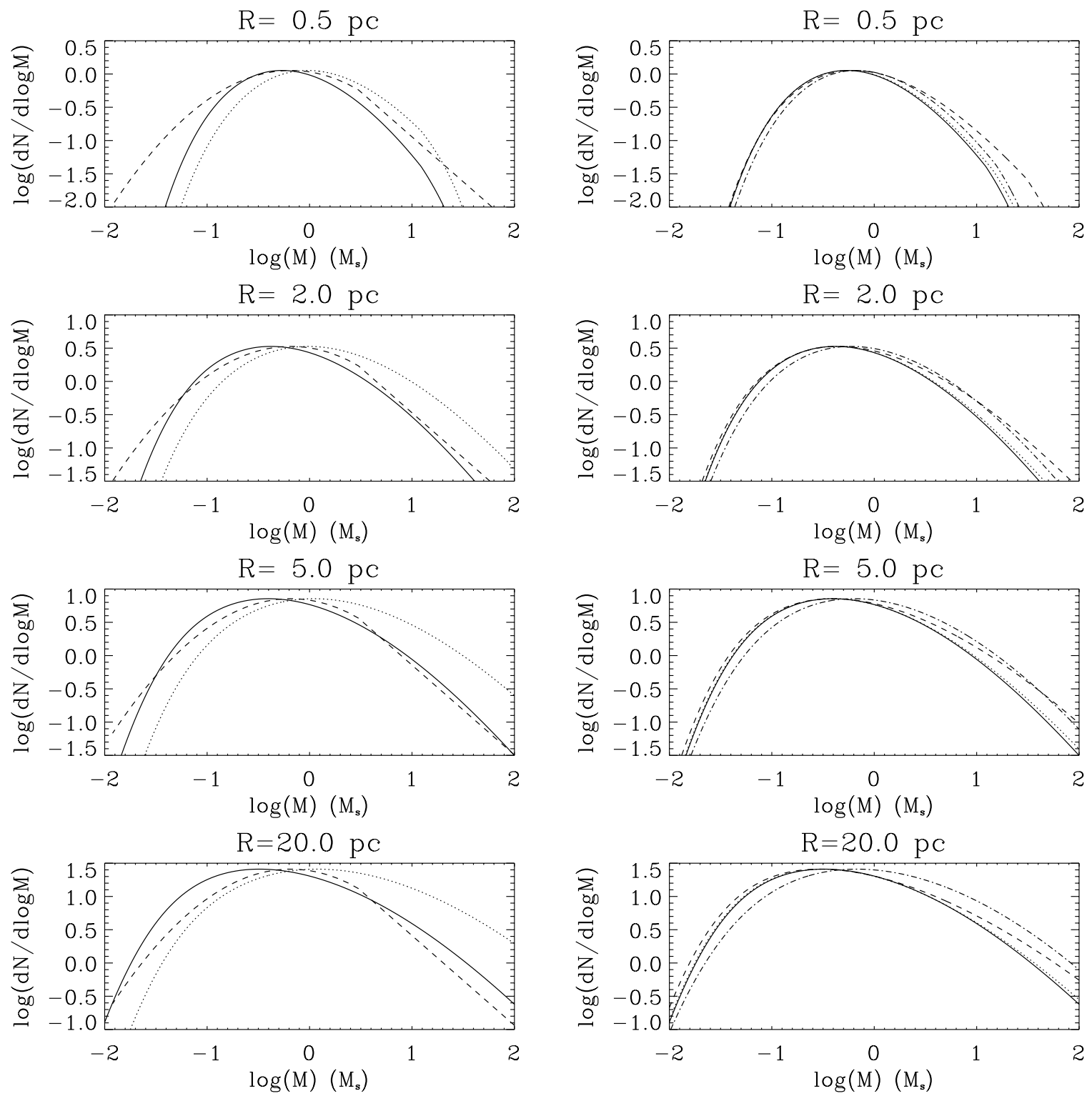

Figure 2. Barotropic and magnetized mass spectra for clumps of size $R_{c}=0.5, R_{c}=2, R_{c}=5$, and $R_{c}=20 \mathrm{pc}$. The clump density and Mach numbers are given by Larson-type relations (Equation (35)) with $u_{0}=1$ and $d_{0}=5,4,3,2$ from top to bottom. Left panel: the solid line corresponds to the time-dependent CMF while the dotted line represents the time-independent one. The dashed line is the Chabrier IMF shifted by a factor of three in mass (see the text). Right panel: the solid line is identical to the left panel. The dotted line corresponds to $V_{a}^{0}=1, \gamma_{b}=0.1$; the dashed line to $V_{a}^{0}=3, \gamma_{b}=0.1$; and the dot-dashed one to $V_{a}^{0}=1, \gamma_{b}=0.3$.

21995, and $355607 M_{\odot}, \bar{n}=8120,2460,970$, and $240 \mathrm{~cm}^{-3}$. As in Figure 1, solid lines display the time-dependent case, dotted lines the time-independent one, while the dashed line corresponds to the SCIMF. In the four cases, except possibly for the smallest clump (as expected; see Section 6.2.2 of Paper II and Section 3.2.2.1 below), the agreement with the SCIMF is very good. Importantly enough, the clump densities found to yield a good agreement with the SCIMF are smaller by a factor of about two to five than what has been found in the timeindependent case for similar agreement (Figure 8 of Paper II), and thus agree fairly well with the "standard" Larson density normalization values, $d_{0} \simeq 3$. This is a direct consequence of the peak position being shifted toward smaller masses, as discussed earlier. Another noticeable improvement is the width of the distribution, which is larger than for the isothermal cases presented in Figure 1. As discussed in Paper II, this improvement stems from the larger compressibility of the gas for a softer than isothermal eos, promoting small-scale collapsing structures, and from the strong dependence of the peak position on $\gamma$ (see Figure 3 of Paper II), which becomes even more acute in the timedependent CMF, whose peak is shifted toward smaller masses compared with the time-independent solution.

\subsubsection{Influence of the Magnetic Field on the Core Mass Function}

To study the influence of the magnetic field on the mass spectrum, we define

$$
V_{a}=V_{a}^{0} \frac{B_{0}^{\mathrm{ref}}}{\sqrt{4 \pi \bar{\rho}}}\left(\frac{\bar{n}}{1000 \mathrm{~cm}^{-3}}\right)^{\gamma_{b}},
$$

where $B_{0}^{\text {ref }}$ is a reference magnetic intensity equal to $10 \mu \mathrm{G}, \bar{n}$ is the cloud mean particle density, and $\bar{\rho}=m_{p} \bar{n}$. We consider four cases. The first one is purely hydrodynamical and serves as a reference. The second one uses $\gamma_{b}=0.3$ and $V_{a}^{0}=1$. The third and fourth ones use $\gamma_{b}=0.1$ and $V_{a}^{0}=1$ or $V_{a}^{0}=3$, respectively. We primarily investigate values of $\gamma_{b}$ smaller 
than 0.5 because our analysis consists of analyzing the density fluctuations generated by supersonic turbulence and identifying the ones that are self-gravitating and that subsequently will be amplified by gravity. Therefore, at the stage where the analysis is performed, the correlation between magnetic intensity and density is the result of turbulent processes rather than gravity, implying $B \propto \rho^{0.1-0.3}$. Note that as emphasized in Hennebelle \& Chabrier (2008), the case $\gamma_{b}=0.5$ is formally equivalent to the isothermal case and all the magnetic results can be obtained by a simple renormalization of the rms velocity and Mach number (see Equation (16)).

The impact of the magnetic field on the mass spectrum can be inferred from a comparison between the various magnetized cases and the hydrodynamical one (Figure 2, right panel). When $V_{a}^{0}=1, \gamma_{b}=0.3$ (dot dashed line), the mass spectrum is essentially shifted toward larger masses with respect to the purely hydrodynamical case. This stems from the magnetic support, as expressed by Equation (18), which adds up to the thermal pressure. Indeed, for this value of $\gamma_{b}$, the magnetic pressure density dependence, $P_{\text {mag }} \propto \rho^{0.6}$, is very similar to the thermal pressure density dependence, in particular, because the Larson eos has an effective exponent at low density which is equal to 0.7 .

When $\gamma_{b}=0.1$, the impact of the magnetic field is different. For $V_{a}^{0}=1$ (dotted line), the departure from the hydrodynamical case (solid line) is only marginal. This is because the magnetic support increases very slowly with density; thus, at high density (which corresponds to low-mass cores), the magnetic support is less important than the thermal one. At high masses, on the other hand, the support is dominated by turbulence. For $V_{a}^{0}=3$ (dashed line), the distribution tends to be shallower at high masses. As discussed in Paper II, the exponent of the mass distribution at high masses, $\mathcal{N}(M) \propto M^{-(1+x)}$, when only pressure terms are included $\left(\mathcal{M}_{\star}=0\right)$, is given by (see Equation (32) of Paper II) $1+x=(9-6 \gamma) /(4-3 \gamma)$, so that if $\gamma<0.2$ the slope becomes shallower than the Salpeter value.

Overall, for a typical magnetic field intensity in the diffuse (low-density) gas of the order of $B \simeq 10 \mu \mathrm{G}$, and a density dependence such that $\gamma_{b}<0.3$, the mass spectrum is not very different from the hydrodynamical case. For $\gamma_{b}=0.3$, the CMF still resembles the SCIMF but is shifted toward larger masses, a direct consequence of the magnetic support, which shifts the characteristic Jeans mass to larger values. When the magnetic field intensity is significantly larger than the aforementioned value (e.g., $30 \mu \mathrm{G}$, like in the considered example), however, the mass spectrum becomes too shallow at large masses and starts departing significantly from a Chabrier/Salpeter IMF in this regime. Therefore, one of the predictions of our theory is that diffuse magnetized environments with magnetic field intensities largely exceeding about $10 \mu \mathrm{G}$ should have high-mass IMF tails shallower than the Salpeter value.

\section{STAR FORMATION RATE}

In this section, we derive the SFR from our time-dependent analytical theory of star formation described in the previous section, analyze its dependence upon various parameters and clump properties, and compare the results with recent observational determinations.

\subsection{Theoretical Considerations}

Following Krumholz \& McKee (2005, KMK), we define the dimensionless star formation rate per free-fall time, $\mathrm{SFR}_{\mathrm{ff}}$, as the fraction of clump mass converted into stars per clump mean free-fall time, i.e., the free-fall time defined at the clump mean density $\bar{\rho}$ :

$$
\mathrm{SFR}_{\mathrm{ff}}=\frac{\dot{M}_{*}}{M_{c}} \tau_{\mathrm{ff}}^{0},
$$

where $\dot{M}_{*}$ denotes the total $S F R$ arising from a clump of mass $M_{c}$ and volume $V_{c} \simeq R_{c}^{3}$. The star formation efficiency (SFE) is defined as the global mass fraction of a clump converted into stars during the lifetime $\tau_{0}$ of the clump, which can last a few free-fall times (e.g., Murray 2011):

$$
\mathrm{SFE}=\frac{M_{*}}{M_{c}}=\mathrm{SFR}_{\mathrm{ff}} \times\left(\frac{\tau_{0}}{\tau_{\mathrm{ff}}^{0}}\right) .
$$

According to these definitions, $\mathrm{SFR}_{\mathrm{ff}}$ is thus given by the integral of the mass spectrum specified by Equation (2) as

$$
\begin{aligned}
\mathrm{SFR}_{\mathrm{ff}}= & \epsilon \int_{0}^{M_{\mathrm{cut}}} \frac{M \mathcal{N}(M) d M}{\bar{\rho}} \\
= & \epsilon \int_{0}^{M_{\mathrm{cut}}} d M \frac{d R}{d M}\left(-\frac{d \delta_{R}}{d R} \frac{\tau_{\mathrm{ff}}^{0}}{\tau_{R}} \exp \left(\delta_{R}\right) \mathcal{P}_{R}\left(\delta_{R}\right)\right. \\
& \left.+\int_{\delta_{R}}^{\infty} \frac{d}{d R}\left[\mathcal{P}_{R}\left(\frac{\tau_{\mathrm{ff}}^{0}}{\tau_{R}}\right)\right] e^{\delta_{R}} d \delta\right) .
\end{aligned}
$$

In this expression, $\epsilon$ is the (supposedly uniform) efficiency with which the mass within the collapsing prestellar cores is converted into stars. ${ }^{9}$ Indeed, during the collapse, a substantial fraction of the mass initially within the core is blown away by outflows and jets. Calculations (e.g., Matzner \& McKee 2000; Ciardi \& Hennebelle 2010), as well as observations, suggest that

$$
\epsilon \simeq 0.3-0.5 \text {. }
$$

Note that Federrath \& Klessen (2012) estimated $\epsilon \simeq 0.3-0.7$ by comparing numerical simulations with observations. In Equation (40), $M_{\text {cut }}$ corresponds to the mass of the largest star that can possibly form in the cloud. Equivalently, according to the mass-scale relation given by Equations (19) or (28), one can define $y_{\text {cut }}=R / R_{c}$, which denotes the largest size fluctuations that can turn unstable in the cloud. The value of $M_{\text {cut }}$ is crucial in setting $\mathrm{SFR}_{\mathrm{ff}}$. Indeed, as emphasized in Paper I (Section 5.1.3), the integral $V_{c} \int_{0}^{\infty} M \mathcal{N}(M) d M$ is equal to the mass of the system itself because integrating up to infinity implies that all pieces of gas, including the very diffuse ones, are Jeans unstable. Therefore, restricting the integration to a finite value implies that any piece of fluid that is not dense enough, i.e., whose density corresponds to a Jeans length larger than a significant fraction of the cloud's size, is excluded from the mass spectrum of collapsing structures. The immediate consequence of such a truncation of the integral is obviously to reduce the SFR.

If $R_{c}$ is the clump's radius, more generally its characteristic scale, then the question is thus to determine up to which fraction of this scale or up to which fraction of the clump mass, the integration should be performed? A similar question concerns the behavior of the density power spectrum whose scale dependence is characterized by $\sigma^{2}(R)$ (Equation (5)). While in the inertial domain, it is well established that the power

\footnotetext{
9 Note the typo in Equation (7) of Hennebelle \& Chabrier (2011b) where it should simply be $d M$ and not $d M / M$ in the integrand.
} 
spectrum of $\log (\rho)$ exhibits a power-law behavior (Beresnyak et al. 2005; Federrath et al. 2010; Audit \& Hennebelle 2010), but this may not be the case when the scale $R$ is approaching the clump size $R_{c}$. This question is directly related to the very definition of the clump itself and how it connects to the surrounding medium. Unfortunately, these questions are far from being settled. Finally, as discussed in Paper I, the second term of the right-hand side in Equation (40) is important only when $y_{\text {cut }} \rightarrow 1$. Therefore, given all the uncertainties when approaching this limit and because the influence of this second term remains limited, we elected to drop it in the rest of the calculations. In that case, after proper normalization, Equations (10), (12), (11), and (40) yield

$$
\begin{aligned}
\operatorname{SFR}_{\mathrm{ff}}^{0}= & -\int_{0}^{\widetilde{M}_{\mathrm{cut}}}\left(\frac{\widetilde{M}_{R}^{c}}{\widetilde{R}^{3}}\right) \frac{d \widetilde{R}}{d \widetilde{M}_{R}^{c}} \frac{d \delta_{R}^{c}}{d \widetilde{R}} \frac{1}{\sqrt{2 \pi \sigma^{2}}} \\
& \times \exp \left(-\frac{\left(\delta_{R}^{c}\right)^{2}}{2 \sigma^{2}}-\frac{\sigma^{2}}{8}\right) d \tilde{M},
\end{aligned}
$$

with

$$
\mathrm{SFR}_{\mathrm{ff}}=\left(\frac{\epsilon}{\phi_{t}}\right) \times \mathrm{SFR}_{\mathrm{ff}}^{0} .
$$

For the case of an isothermal eos, for which the mass-size relationship is given by Equation (19), this yields

$$
\begin{aligned}
\operatorname{SFR}_{\mathrm{ff}}^{0}= & -2 \int_{0}^{\widetilde{M}_{\mathrm{cut}}} \frac{1}{\widetilde{R}^{3}} \frac{1+(1-\eta) \mathcal{M}_{*}^{2} \widetilde{R}^{2 \eta}}{\left[1+(2 \eta+1) \mathcal{M}_{*}^{2} \widetilde{R}^{2 \eta}\right]} \\
& \times\left(\frac{\widetilde{M}}{\widetilde{R}^{3}}\right)^{-\frac{1}{2 \sigma^{2}} \ln \left(\frac{\tilde{M}}{\widetilde{R}^{3}}\right)} \times \frac{e^{\left(-\frac{\sigma^{2}}{8}\right)}}{\sqrt{2 \pi} \sigma} d \tilde{M} .
\end{aligned}
$$

Conversely, the SFR can also be derived from Equation (40) (still dropping the second term) as

$$
\begin{aligned}
\operatorname{SFR}_{\mathrm{ff}}^{0} & =\int_{\widetilde{\delta}_{\text {cut }}}^{\infty} \frac{\tau_{\mathrm{ff}}^{0}}{\tau_{R}\left(\delta_{R}\right)} e^{\delta_{R}} \mathcal{P}\left(\delta_{R}\right) d \delta_{R} \\
& =\int_{\widetilde{\rho}_{\mathrm{cut}}}^{\infty} \widetilde{\rho}^{1 / 2} \mathcal{P}(\widetilde{\rho}) d \widetilde{\rho} \\
& =\frac{1}{2} e^{\left(\frac{3 \sigma^{2}}{8}\right)}\left[1+\operatorname{erf}\left(\frac{\sigma^{2}-\ln \left(\widetilde{\rho}_{\mathrm{cut}}\right)}{2^{1 / 2} \sigma}\right)\right] .
\end{aligned}
$$

We stress that this is possible only because the second term in Equation (40) has been dropped. The value of $\tilde{\rho}_{\text {cut }}$ in Equation (45) can be derived from the collapse condition derived in our theory (see Equation (29) of Paper I), namely, $\tilde{\rho}=e^{\delta_{R}}>e^{\delta_{R}^{c}}$, or similarly from the mass-size relations given by Equations (19) or (28). For the isothermal case, this simply yields

$$
\widetilde{\rho}_{\text {cut }}=\widetilde{R}_{\text {cut }}^{-2}\left(1+\mathcal{M}_{\star}^{2} \widetilde{R}_{\text {cut }}^{2 \eta}\right),
$$

where $\widetilde{R}_{\text {cut }}=\left(y_{\text {cut }} R_{c} / \lambda_{J}^{0}\right)$. As clearly expressed by this equation, physically, $\widetilde{\rho}_{\text {cut }}$ is the minimum density for which a perturbation, whose associated total (thermal + turbulent) Jeans length is equal to a maximum fraction $y_{\text {cut }}$ of the clump's size, can lead to a gravitational instability. Indeed, Equation (46) can be rewritten as follows:

$$
\left(\lambda_{J}\right)_{\mathrm{cut}}=\frac{y_{\mathrm{cut}} R_{c}}{\left[1+\mathcal{M}_{\star}^{2}\left(\frac{y_{\mathrm{cut}} R_{c}}{\lambda_{J}^{0}}\right)^{2 \eta}\right]^{1 / 2}} .
$$

It is important to stress that this procedure differs not only quantitatively but also qualitatively from the ones defined in KMK and Padoan \& Nordlund (2011, PN). In these author formalisms, the critical density corresponds to a new, arbitrary scale, respectively, a sonic scale or a shock scale, characteristic of a process supposed to be necessary for the collapse. In contrast, in our formalism, the only relevant scale is the size of the cloud itself. So, even though this can be expressed as a density in Equation (45), the proper way to look at it, as clearly expressed by Equation (47), is in terms of the maximum size of the fluctuations for a given cloud's size and density, which are able to grow and lead to gravitational instability. Moreover, in the KMK and PN formalisms, the critical density is proportional to $\mathcal{M}^{2}$, implying that only very dense structures will lead to star formation. In contrast, in our formalism, any structure of any density can collapse if its gravitational energy dominates over all sources of support, as long as the associated perturbation can grow and become unstable. In practice, our results do not depend significantly on the value of $y_{\text {cut }}$, except when $y_{\text {cut }} \rightarrow 0$ and for low Mach numbers (see next section). Indeed, $y_{\text {cut }}$ only affects the limit of the integration, which corresponds to a regime where the (lognormal) PDF is small.

As defined above, $\mathrm{SFR}_{\mathrm{ff}}^{0}$ represents the SFR calculated from our theory for a core-to-star mass conversion efficiency $\epsilon=1$ during the collapse, and for a characteristic time within which new mass reservoirs $\widetilde{M}$ of scale $\widetilde{R}$ become gravitationally unstable, i.e., new cores are produced, equal to one free-fall time at the core's density, i.e., $\tau_{R}=\tau_{\mathrm{ff}}(R)\left(\phi_{t}=1\right)$. In that sense, $\mathrm{SFR}_{\mathrm{ff}}^{0}$ represents the "core formation rate" per free-fall time. As already mentioned, observations and simulations point to $\epsilon \simeq 0.3-0.5$, while our estimate of $\tau_{R}$ in Section 2.2.2 leads to $\phi_{t} \simeq 3$. Consequently, we typically have

$$
\frac{\mathrm{SFR}_{\mathrm{ff}}}{\mathrm{SFR}_{\mathrm{ff}}^{0}} \simeq 0.1-0.2 .
$$

\subsection{Results: Dependence upon Clump Properties}

\subsubsection{Models and Assumptions}

A detailed comparison with the theoretical SFRs derived by $\mathrm{KMK}$ and $\mathrm{PN}$ was presented in $\mathrm{HC} 11$, and thus will not be repeated here. As mentioned in this paper, the key differences between these two theories and the present one are twofold. First, both the KMK and PN theories assume that the SFR is determined by one single typical free-fall time, defined as the free-fall time at the cloud mean density, $\tau_{\mathrm{ff}}^{0}$. Given the very clumpy nature of molecular clouds and the enormous range of density fluctuations present within these entities, defining one single mean free-fall time for star formation seems to be hardly justified. This point becomes particularly acute given the fact that there is no evidence that most of the regions within molecular clouds are collapsing, and if so that they are collapsing at $\tau_{\mathrm{ff}}^{0}$ (see, e.g., Kennicutt \& Evans 2012). The second essential difference resides in the fact that both KMK and PN assume an ad-hoc critical density $\rho_{\text {crit }}$ for star formation to occur (see HC11). Their SFR is thus simply obtained by estimating the gas fraction with density larger than $\rho_{\text {crit }}$. In contrast, in our theory, the free-fall time density dependence of any collapsing structure of density $\rho_{R}$ is properly accounted for, as the crossing time $\tau_{R}$, proportional to $\rho_{R}^{-1 / 2}$, consistently varies with $M$ and $R$. Therefore, a dense core of density $\rho$ collapses in a densitydependent free-fall time $\tau_{\mathrm{ff}}(\rho)$, which can differ significantly from the clump's mean free-fall time. In our theory, there is 
no particular scale or critical density, as we sum up over all gravitationally unstable overdense regions, whatever their scale or density is (see Equations (1) and (2)). This is indeed expected if the density fluctuations are induced by turbulence, which is by essence a multi-scale process, and by the fact that any piece of fluid can collapse if its (turbulent+thermal) kinetic energy is dominated by its gravitational energy.

Comparisons between Equations (42) and (45) and the SFRs of KMK and PN have been presented in $\mathrm{HC} 11$ and will not be repeated here. It was shown in this paper that the KMK SFR differs by more than one order of magnitude from the others, underestimating the observed SFR by a similar amount, as already noticed by Heiderman et al. (2010). The PN SFR and those given by Equations (42) and (45) agree within a factor $\sim 2-3$, provided the $\left(\epsilon / \phi_{t}\right)$ factor is properly included in the PN relation (see HC11). The main reason why the present determinations yield a larger SFR than $\mathrm{KMK}^{10}$ and $\mathrm{PN}$ is that when properly accounting for the density dependence of the collapsing structure free-fall times, denser fluctuations, in particular the ones denser than a given arbitrarily defined density $\rho_{\text {crit }}$, collapse faster than lower density ones, leading to a larger formation of low-mass cores. Although qualitatively similar to the PN results, ours still differ from these latter (see Figure 1 of HC11). Indeed, while $\mathrm{SFR}_{\mathrm{ff}}^{0}$ tends to decrease steadily with increasing virial parameter, $\alpha_{\mathrm{vir}}=2 E_{\mathrm{kin}} / E_{\mathrm{grav}}$, in the PN theory, it exhibits a more flattish (nearly constant) behavior before decreasing more steeply at high $\alpha_{\text {vir }}$ in our case, as will be examined in detail below. The reason stems from the gas that is more diffuse than $\rho_{\text {crit }}$ not being taken into account in the PN model while being accounted for, if indeed collapsing, in our model. As mentioned above, in our theory, if a piece of fluid of even very low density (e.g., smaller than $\rho_{\text {crit }}$ in PN) has a size significantly larger than the (thermal or turbulent) Jeans length at its density, it is subject to collapse and thus must be taken into account. This point will be crucial when comparing to SFR observational determinations at low density (Section 4). As mentioned earlier, this is a fundamental difference between our theory and both the KMK and PN theories.

\subsubsection{Star Formation Rate: Results}

We now examine the dependence of our theoretical SFR upon various clump characteristic parameters and physical properties.

3.2.2.1. Dependence upon the clump's large-scale cutoff, size, and density. Figure 3 portrays $\mathrm{SFR}_{\mathrm{ff}}^{0}$, for the non-isothermal case, for $b=0.5$ (left column) and $b=0.25$ (right column) in Equation (4), as a function of the cutoff parameter, $y_{\text {cut }}=R / R_{c}$, for various clump sizes and mean densities (this means that the parameter $d_{0}$, which determines the density normalization at $1 \mathrm{pc}$ in the Larson relation (Equation (35)), is constant along each line but varies from one line to another). As mentioned earlier and expected from the truncation of the integral in Equation (42), $\mathrm{SFR}_{\mathrm{ff}}^{0}$ is basically independent of $y_{\text {cut }}$ for $y_{\text {cut }} \gtrsim 0.3$ but quickly drops as this parameter approaches very small values. As also expected, the strong decrease of $\mathrm{SFR}_{\mathrm{ff}}^{0}$ occurs at higher $y_{\text {cut }}$ values for smaller, less massive clumps and the truncation becomes more drastic as density decreases. Indeed, as already noticed in Papers I and II (see Figure 8 of Paper II), the core

\footnotetext{
10 Besides the fact that KMK further assume that the critical free-fall time is equal to the free-fall time of the cloud, which implies that $\widetilde{\rho}_{\text {crit }}=1$, a very consequential issue, as shown in HC11.
}

mass spectrum is truncated both at high and small masses compared with the observationally determined CMF/IMF for small ( $\lesssim 0.5 \mathrm{pc})$ clumps, with a drastically reduced CMF for low-density clumps. This stems from two reasons: (1) the scale dependence, based on Larson's relations, of both the global and local Mach numbers, $\mathcal{M}$ and $\mathcal{M}_{\star}$, which enter our formalism (see Equations (16) and (20)) and are responsible for generating and stabilizing the initial density fluctuations leading eventually to prestellar cores. The smaller the clump, the smaller these values and thus the narrower the core mass spectrum (see Paper I). (2) The fact that for small clumps or low-density clumps, the Jeans scale becomes comparable to or larger than the size of the clump itself (see Equation (14)), inhibiting gravitational collapse.

According to these results, star formation is thus predicted to occur dominantly in the largest, most massive clumps and/or in the densest (parts of) clumps, a conclusion indeed supported by observations. As seen in the figure, for densities $\bar{n} \gtrsim 2000 \mathrm{~cm}^{-3}$ and for $y_{\text {cut }} \gtrsim 0.1-0.3$, depending on the clump's size, the SFR depends only weakly on $y_{\text {cut }}$ and reaches values in the range $\mathrm{SFR}_{\mathrm{ff}}^{0} \simeq 0.3-3.0$, depending on the size of the clump, for the $b=0.5$ case. The fact that $\mathrm{SFR}_{\mathrm{ff}}^{0}$, the SFR per free-fall time, can be larger than 1 is due to its density dependence. Indeed, $\mathrm{SFR}_{\mathrm{ff}}^{0}$ is defined with respect to $\tau_{\mathrm{ff}}^{0}$, the free-fall time at the cloud's mean density, but fluctuations of size $R$ whose density is larger than $\bar{\rho}$ collapse in a shorter time. As has already been discussed, the value of $y_{\text {cut }}$ beyond which no star can form is ill-determined and may depend upon cloud parameters. In the rest of our calculations, we will assume $y_{\text {cut }} \simeq 0.1-0.3$ (see previous section), which means that only perturbations whose size is at most of the order of about one-tenth to one-third of the cloud's size are relevant to produce gravitationally bound prestellar cores. As just mentioned, for larger values of $y_{\text {cut }}$, the SFR remains basically unchanged.

As seen from Figure 3, low-density clumps $\left(\bar{n} \lesssim 1500 \mathrm{~cm}^{-3}\right)$ smaller than about $\sim 2$ pc yield quite small or even negligible values of $\mathrm{SFR}_{\mathrm{ff}}^{0}$, except for values of $y_{\text {cut }}$ approaching unity, a rather unlikely possibility. Phrased differently, at low density, very large clumps, but only very large clumps, still contribute appreciably to star formation. As mentioned in the previous section, this is one of the direct consequences of our theory and cannot be the case in a theory arbitrarily defining a density threshold for star formation, a point of importance when we will compare with observational determinations (Section 4). As just mentioned, star formation should thus occur dominantly in dense enough (regions of) clumps or in very large and massive clumps and to increase with clump density and clump mass/size. It is worth stressing that this strong dependence upon density or mass for efficient star formation naturally arises in our formalism and does not stem from an ad-hoc threshold condition for star formation.

Finally, the figure shows the dependence of the SFR upon the coefficient $b$ entering Equation (4), which relates the rms Mach number to the width of the density PDF, $\sigma_{0}$ (see Equation (4)). Although the results are qualitatively similar, globally, $\mathrm{SFR}_{\mathrm{ff}}^{0}$ is smaller by a factor of a few for large values of $y_{\text {cut }}$ and drops drastically below larger values of $y_{\text {cut }}$ for $b=0.25$ than for $b=0.5$. Indeed, as shown in detail in Section 3.1 of Paper II, the mass spectrum peaks at larger masses and the density PDF gets narrower for smaller values of $b$ because the gas is less compressible. Since the respective contributions of the turbulence forcing mechanisms (and thus the value of $b$ ) in molecular clouds are rather ill-determined and may vary 

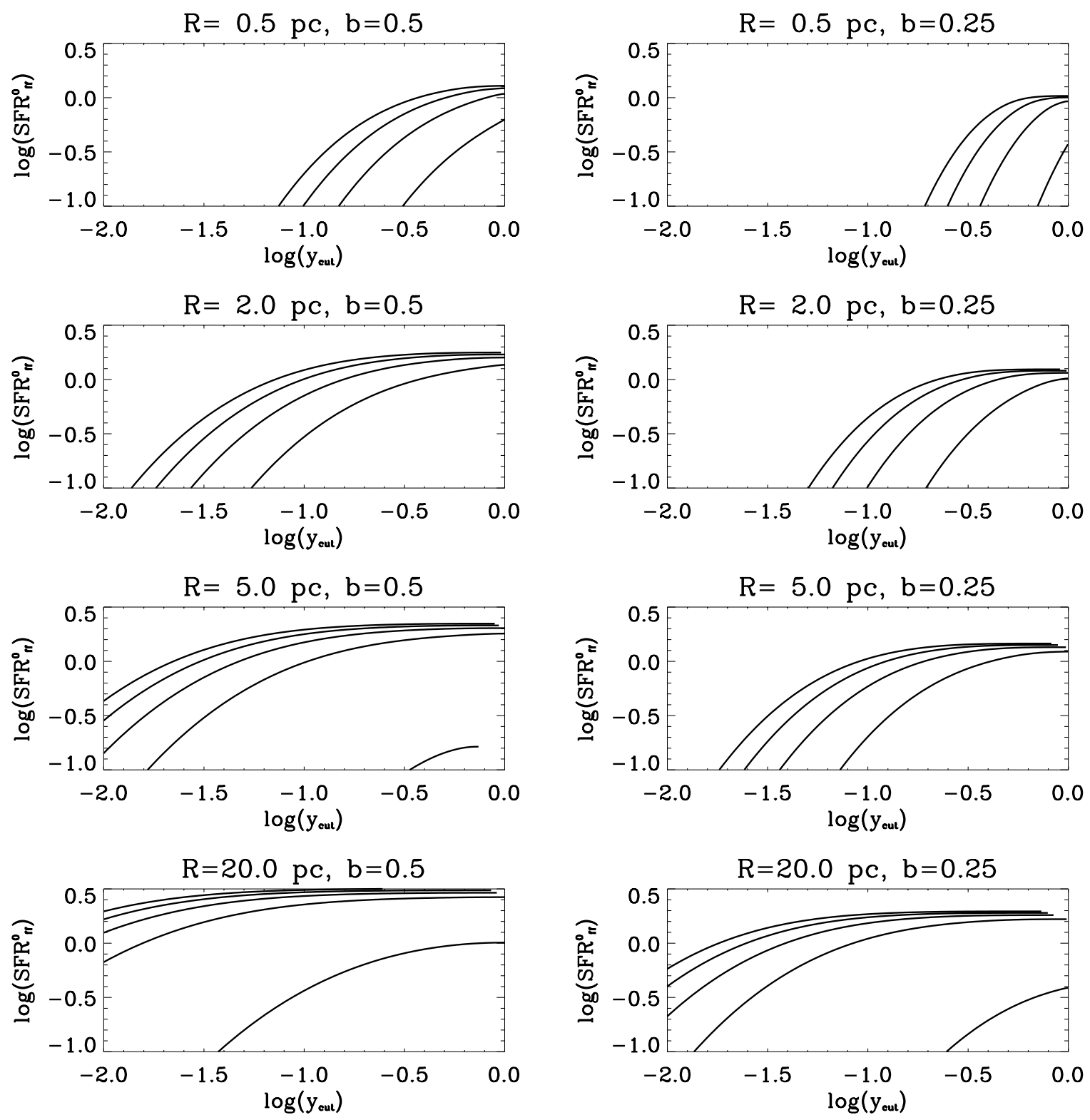

Figure 3. Value of $\mathrm{SFR}_{\mathrm{ff}}^{0}$ for various clump sizes and densities, namely (from left to right), $d_{0}=4.5,3,1.5,0.7,0.1$ in the non-isothermal case. Left column: $b=0.5$; right column: $b=0.25$.

from cloud to cloud, depending for instance on the presence of expanding $\mathrm{H}$ II regions around massive stars or supernova explosions, this implies significant possible variations of the SFR, depending on the environment.

3.2.2.2. Dependence upon the virial parameter. The dependence of the SFR upon the characteristic virial parameter $\alpha_{\text {vir }}$ of a clump of radius $R_{c}=L_{c} / 2$ and mean density $\bar{\rho}$, $\alpha_{\mathrm{vir}}=2 E_{K} / E_{G}=5 V_{\mathrm{rms}}^{2} /\left(\pi G \bar{\rho} L_{c}^{2}\right)$, which measures the ratio of turbulence over gravitational energy within the clump, has been examined in HC11 in the isothermal case and will be examined in the next subsection in the non-isothermal one. It is illustrated in Figure 4, which displays the values of $\mathrm{SFR}_{\mathrm{ff}}^{0}$ for various clump sizes/masses, for three typical Mach numbers, $\mathcal{M}=16$ (top), 9 (middle), and 4 (bottom). As a general trend, as discussed in $\mathrm{HC} 11$, increasing $\alpha_{\text {vir }}$ leads to a decrease of the SFR, with an abrupt reduction above some typical value of $\alpha_{\text {vir }}$, which depends on the Mach number. This stems from the fact that as $\alpha_{\text {vir }}$ increases, the increasing contribution of kinetic energy over potential energy prevents gravitational collapse and thus inhibits star formation. As increasing $\alpha_{\text {vir }}$ implies decreasing the clump's mass/size, as $\alpha_{\mathrm{vir}} \propto L_{c}^{-2} \propto M_{c}^{-2 / 3}$ (at fixed Mach number and density), star formation is thus highest in the largest and most massive clumps, which correspond to the smallest values of $\alpha_{\mathrm{vir}}$, in agreement with the conclusion reached in the previous subsection. Giant molecular clouds (GMCs) in the Milky Way, with masses in the range $\sim 10^{3}-10^{6} M_{\odot}$, are generally weakly gravitationally bound structures, with virial parameters in the range $\alpha_{\text {vir }} \approx 0.3-3$, with slightly decreasing $\alpha_{\text {vir }}$ with increasing cloud mass, as just noted (Heyer et al. 2009; Murray 2011). Accordingly, the rather low observed SFR values might partly stem from the fact that most molecular clouds have in general virial parameter values slightly above unity, i.e., they depart from perfect virialization, being held together partly by the confining ram pressure of turbulent flows in the interstellar medium (ISM).

3.2.2.3. Thermodynamics of the gas. For the non-isothermal case, we need to specify a temperature-density distribution (see Equation (18) of Paper II). We choose $n_{0}=200 \mathrm{~cm}^{-3}$ and 

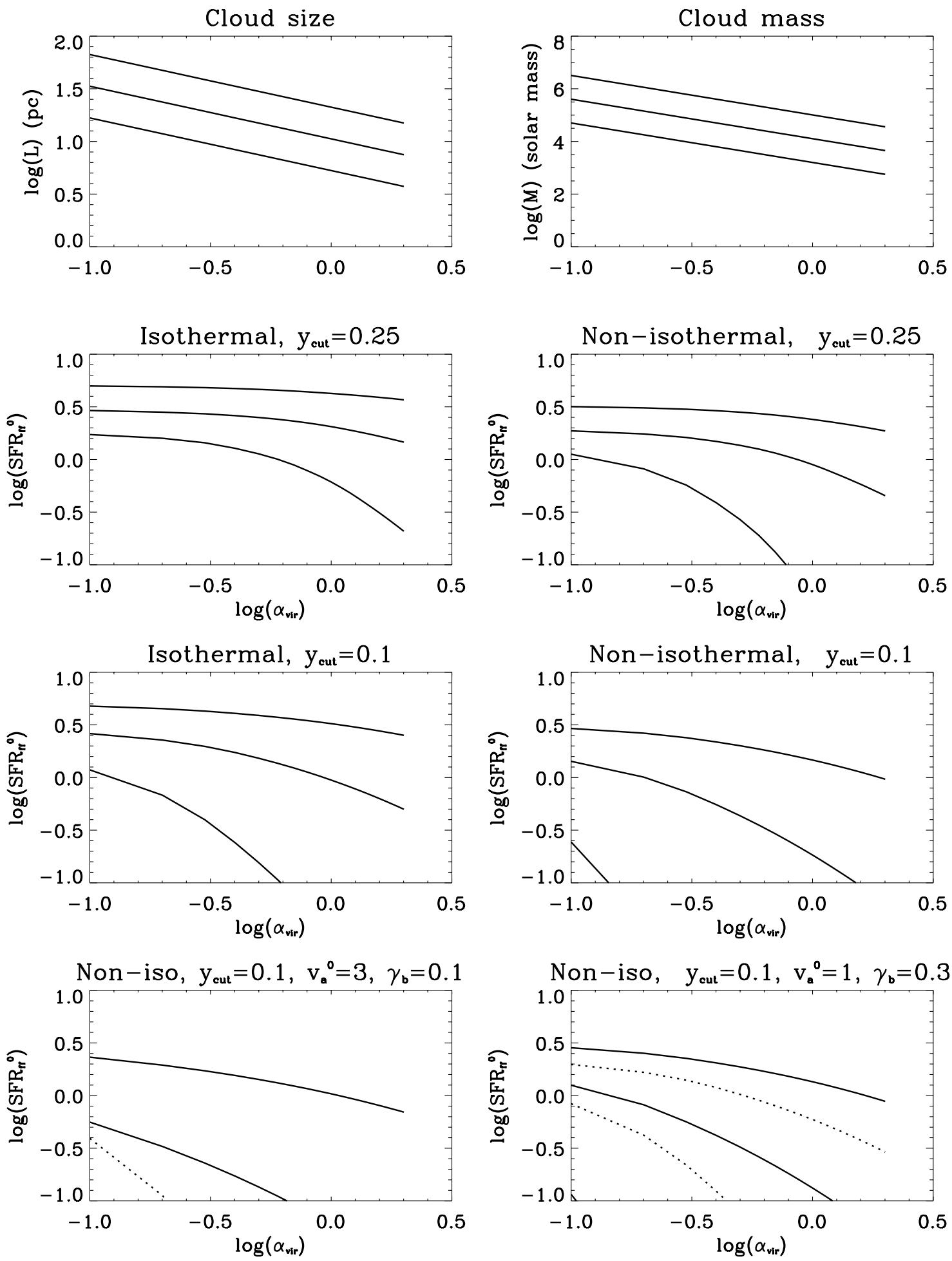

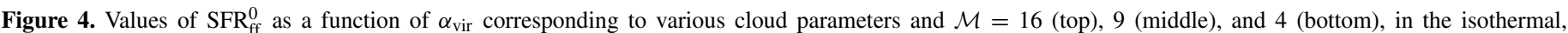

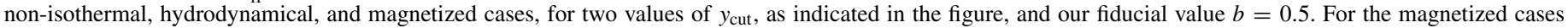

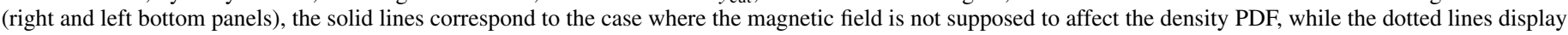
the results when the effect of the field on the width of the PDF is taken into account (see the text).

$T_{0}=20 \mathrm{~K}$ at $10^{4} \mathrm{~cm}^{-3}$. Assuming that the velocity dispersion remains unchanged, we recalculate accordingly the speed of sound and the Mach number in the non-isothermal case (see Equation (20) of Paper II).

As seen in Figure 4, taking into account the thermodynamics of the gas yields values of $\mathrm{SFR}_{\mathrm{ff}}^{0}$, for the same clump conditions, smaller by a factor of about $\sim 1.5-2$ than the isothermal case in the nearly constant $\mathrm{SFR}_{\mathrm{ff}}^{0}$ region, which corresponds to small values of $\alpha_{\text {vir }}$, but leads to a significantly steeper decrease for increasing values of the virial parameter, in particular for $\alpha_{\text {vir }} \gtrsim 1$. As explored in detail in Paper II, this is a direct consequence of the density dependence of the Mach number for non-isothermal gas. Indeed, when taking into account the thermodynamics of the gas, the clump is warmer at low densities, 
which implies lower Mach numbers than for the isothermal case (see Paper II), decreasing the SFR, as seen in the figure.

As seen in the figure, for low Mach numbers that correspond to small-size clouds, the value of $y_{\text {cut }}$ has a significant impact on $\mathrm{SFR}_{\mathrm{ff}}^{0}$, as already discussed in Section 3.2.2.1, leading to substantial uncertainty on the SFR for such low-mass/smallsize clumps. Fortunately, such clumps are predicted to contribute almost negligibly to star formation, as mentioned earlier.

3.2.2.4. The role of magnetic field. The two bottom panels of Figure 4 show the SFR in the two magnetic cases corresponding to $V_{a}^{0}=1, \gamma_{b}=0.3$ and $V_{a}^{0}=3, \gamma_{b}=0.1$, respectively, adopting $y_{\text {cut }}=0.1$.

In order to decipher the contributions of the various physical effects, we have calculated the SFR in the magnetized case, first keeping the same PDF as in the hydrodynamical case (as described by Equation (4)) but then also taking into account the fact that the magnetic field can modify the width of the density PDF, as investigated, for example, in Molina et al. (2012). In a magnetized flow, the dependence upon the Alfvénic Mach number must be taken into account. Based on their numerical simulations, Molina et al. (2012) propose an analytical relation to predict the dependence of the variance of the lognormal density distribution upon the rms Mach number in magnetized supersonic turbulent gas. The result depends on the index $\gamma_{b}$. For $\gamma_{b}=0$, i.e., $B$ independent of the density, they recover the hydrodynamical dependence (Equation (4)). When $\gamma_{b}=0.5$, i.e., $B \propto \sqrt{\rho}$, they find that in the super-Alfvénic case, the variance is given by

$$
\sigma_{0}^{2}=\ln \left(1+b^{2} \mathcal{M}^{2} \frac{\beta}{\beta+1}\right),
$$

where $\beta=P_{\text {th }} / P_{\text {mag }}=2 C_{s}^{2} / V_{a}^{2}$.

The basic effect of the magnetic field on the SFR, when assuming no variation of the density PDF compared with the hydrodynamical case, is illustrated by the solid lines in the bottom left and right panels, to be confronted with the SFR values displayed on the right third panel. As expected, the magnetic field tends to reduce the SFR by a factor of a few. The amplitude of this effect obviously depends on the field intensity, as explained in Section 2.3.3. For $V_{a}^{0}=1, \gamma_{b}=0.1$ (not displayed here), the SFR is identical to the hydrodynamical case, while for $V_{a}^{0}=3, \gamma_{b}=0.1$ (left bottom panel), it is reduced by a factor of less than 2 for $\mathcal{M}=16$ and about 2 for $\mathcal{M}=9$. Stronger values of the magnetic field obviously lead to even lower SFRs. However, the values we used are within the range of the values typically measured in molecular clouds.

The dotted lines of the bottom left and right panels show the SFR when the influence of the magnetic field on the density PDF is taken into account, as described above. With our choice of parameters, we find that at the mean density $\beta=0.036$ for $V_{a}^{0}=3, \gamma_{b}=0.1$ and $\beta=0.6$ for $V_{a}^{0}=1, \gamma_{b}=0.3$. The first value in particular significantly reduces the width of the density PDF. Clearly, the impact of the magnetic field is much more pronounced and the SFR drops by a factor of several. We stress, however, that, as discussed in Molina et al. (2012), the relation given by Equation (49) only holds for $\gamma_{b}=0.5$ and does not work well at small and intermediate Mach numbers. Therefore, there are still considerable uncertainties here due to the lack of exact knowledge of the density PDF in the presence of the magnetic field and also of the precise gas density-magnetic intensity relation.

Finally, we also note that in the presence of a magnetic field, the coefficient $\phi_{t}$ could possibly be further increased, further reducing the SFR. Indeed, it is likely that the magnetic field and the velocity field of the perturbation that eventually gives rise to the gravitationally unstable perturbation must be sufficiently aligned. Otherwise, after a weak density enhancement, the magnetic pressure will probably stop the contraction.

Note that PN and Federrath \& Klessen (2012) find that in their simulations, the SFR is indeed reduced by a factor two to three when the flow is significantly magnetized.

3.2.2.5. The role of turbulence. As in the theories of KMK, PN, and Hopkins (2012a, 2012b), turbulence is at the heart of our star formation theory. We have shown in Paper I (Section 6.3) that in the time-independent version of our IMF theory, turbulence, globally, has a negative impact on star formation. The stronger the turbulence, both at the global clump scale (as formalized by $\mathcal{M}$ ) and the local (Jeans length) scale (as formalized by $\mathcal{M}_{\star}$ ), the smaller the global clump mass fraction encapsulated into gravitationally bound prestellar mass reservoirs. Indeed, although increasing the Mach number increases the width of the PDF, promoting the formation of small-scale structures, it leads to a decrease in the number of collapsing structures around the peak of the CMF (see Papers I and II). As shown in the previous sections, however, star formation will keep processing during the clump's lifetime and time dependence changes the final clump's mass fraction, ending up forming collapsing cores. The impact of turbulence on $\mathrm{SFR}_{\mathrm{ff}}^{0}$ is illustrated in Figure 5. This figure portrays the behavior of $\mathrm{SFR}_{\mathrm{ff}}^{0}$ at fixed cloud density $(\bar{n}=$ constant along each line) for different cloud sizes, as a function of $V_{0}$, and thus of $\mathcal{M}$ (see Equation (16)), spanning a range $\mathcal{M} \simeq 4-30$. The timeindependent results are displayed in Figure 6. In this latter case, the SFR steadily decreases with increasing levels of turbulence, as mentioned above. As shown in Figure 5, however, including time dependence in our formalism significantly changes this behavior, with the SFR now increasing, although modestly, with the level of turbulence. The reason is twofold. First, increasing the Mach number shifts the peak of the CMF toward smaller masses (see Paper I), a behavior exacerbated when time dependence is considered, as discussed in Section 2.2.4. Since small-scale structures have shorter free-fall times $\left(\tau_{R} \propto \rho^{-1 / 2}\right)$, this increases the number of small cores and thus globally increases the SFR (see Equation (40)). Second, massive mass reservoirs, considered to be stable in the static case, can now fragment into small-scale structures as their internal turbulent motions lead to new small-scale overdense regions, globally increasing the number of collapsing cores. This positive impact of turbulence upon the SFR is in agreement with the results of PN but contrasts with those of KMK. As demonstrated in $\mathrm{HC} 11$, this decreasing dependence of the SFR with increasing Mach number in the KMK theory (see their Equation (30)) stems from the missing $\widetilde{\rho}_{\text {crit }}^{1 / 2}$ term in their Equation (20), which stems from the fact that KMK assume that the critical free-fall time is equal to the free-fall time of the cloud. The dependence of the SFR upon $b$ illustrated in Figure 5 has already been addressed previously.

The fact that in the time-dependent formalism, turbulence is found to enhance star formation seems, at first glance, to be in contradiction with earlier numerical simulations (see, e.g., MacLow \& Klessen 2004). However, as discussed in HC11, the behavior we infer for the SFR is qualitatively similar to that inferred by PN (see also Federrath \& Klessen 2012). In their simulations, these authors find that for a given $\alpha_{\text {vir }}$, the SFR increases with the Mach number. The critical point here is that when turbulence is too high (i.e., $\alpha_{\text {vir }}$ is typically 

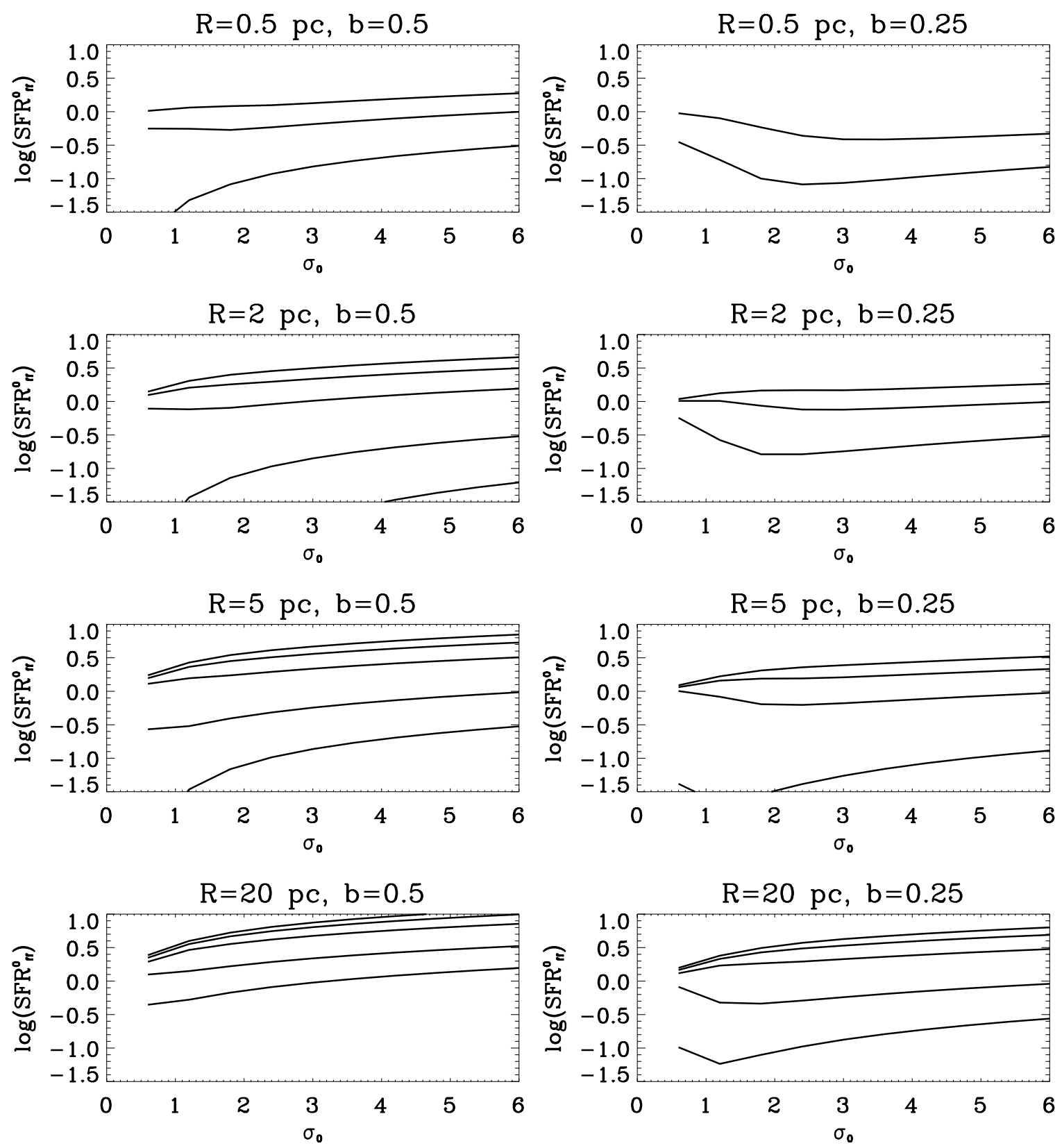

Figure 5. Value of $\mathrm{SFR}_{\mathrm{ff}}^{0}$ as a function of $V_{0}$ for various cloud sizes and densities, namely (from top to bottom), $d_{0}=10,5,2,0.5,0.2$, in the non-isothermal case. Left column: $b=0.5$; right column: $b=0.25$.

larger than 1), the kinetic energy will lead to an expansion of the clump, an effect which is neither included in the present analytical calculations nor in the turbulence in the box numerical simulations of PN. We thus intuitively expect a non-monotonic behavior of the SFR with turbulence. When $\alpha_{\text {vir }}$ is small, turbulence enhances star formation because of the presence of converging motions that lead to density enhancements. On the other hand, if turbulence becomes too large ( $\alpha_{\text {vir }} \gtrsim 1$ or so), then it triggers a fast expansion of the cloud which reduces the density and quenches star formation (note that Vázquez-Semadeni et al. 2003 do see such a non-monotonic behavior with turbulence).

3.2.2.6. Summary of the results. To conclude this section, we summarize the main results of our calculations at this stage. As mentioned above and seen in Figures 4 and 5, for all typical clump conditions we have explored that correspond to clump sizes $\sim 0.5-20 \mathrm{pc}$ and masses $\sim 200-10^{6} M_{\odot}, \mathcal{M}=4-30$, $\mathrm{SFR}_{\mathrm{ff}}^{0}$ lies in the range $\approx 0.01-5$ in the non-isothermal case, even though the value of $y_{\text {cut }}$ significantly affects these values at low Mach. Lower values of SFR $\mathrm{ff}^{0}$ would imply either $y_{\text {cut }}$ of the order of $1 / 30$ or even $1 / 100$, which does not look very realistic, or very small clumps $(\lesssim 0.1 \mathrm{pc})$, for which the mass spectrum is drastically truncated and is completely incompatible with the observed CMF/IMF (see Figure 8 of Paper II). According to our calculations, the value $\mathrm{SFR}_{\mathrm{ff}}^{0} \approx 0.01$ thus seems to be a lower limit for realistic clump conditions. As shown above, the larger SFR values are reached for the densest clumps and the largest/most massive clumps, which correspond to the smaller values of the virial parameter and are thus likely to be gravitationally bound structures. Significant star formation, however, is still predicted to occur in low-density clumps, providing they are large enough (1) to generate enough turbulence levels (high enough Mach numbers) and (2) to largely exceed their typical Jeans mass. We must recall, however, that $\mathrm{SFR}_{\mathrm{ff}}^{0}$ is the SFR obtained assuming that self-gravitating 

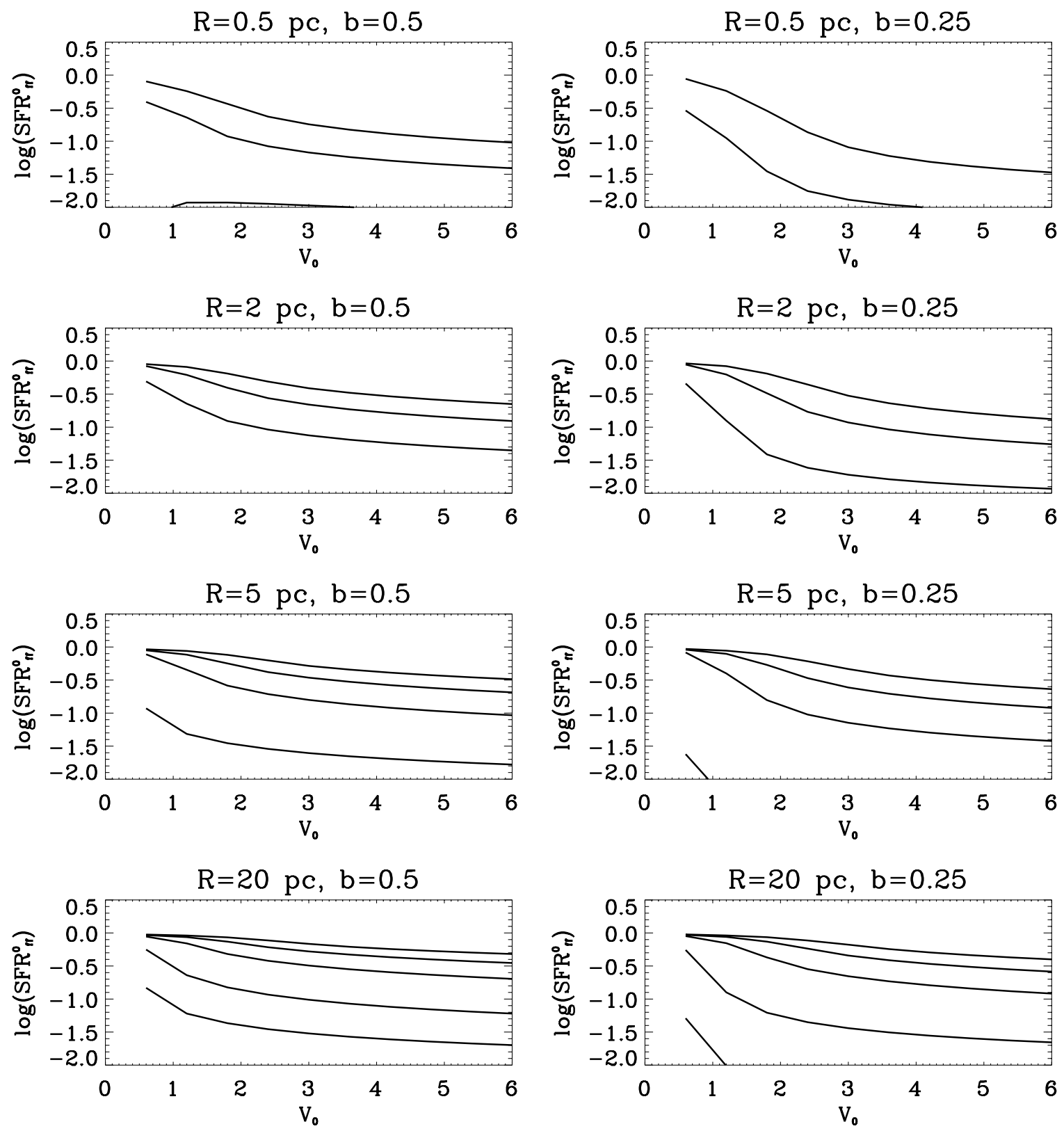

Figure 6. Same as Figure 5 when the free-fall time dependence of the collapsing structures is not taken into account.

fluctuations are replenished within one single free-fall time $\left(\phi_{t}=1\right)$ and with a $100 \%$ efficiency of initial mass reservoir to star conversion during the gravitational collapse $(\epsilon=1)$. $\mathrm{SFR}_{\mathrm{ff}}^{0}$ must thus be multiplied by a factor $\epsilon / \phi_{t}<1$ to yield the effective value $\mathrm{SFR}_{\mathrm{ff}}$. The dependence of these two parameters upon the local physical conditions such as magnetic field intensity or radiative feedback remains very uncertain, but both processes tend to decrease the final star formation rate $\mathrm{SFR}_{\mathrm{ff}}$, either by decreasing $\epsilon$ or by increasing $\phi_{t}$. As mentioned earlier, observational and numerical results suggest that $\epsilon / \phi_{t} \approx 0.1-0.2$. Therefore, according to our calculations, the SFR per free-fall time in dense molecular clumps with typical aforementioned properties is estimated to lie in the range $\mathrm{SFR}_{\mathrm{ff}} \approx 0.001-1.0$, for $y_{\text {cut }}=0.1-0.3$, for virial parameters in the range $0.3 \lesssim \alpha_{\text {vir }} \lesssim 3$, in agreement with typical observed values (see, e.g., Murray 2011 and Section 4 below). The calculations of a mean value of the SFR, integrating over the clump mass spectrum, will be addressed in Section 5.

\section{COMPARISON WITH OBSERVATIONS}

\subsection{Star Formation Rate versus Gas Surface Density}

As seen in the previous section, the SFR per free-fall time can vary by several orders of magnitude depending on the clump's conditions (mass, density, typical Mach number) but also, unsatisfactorily enough, on the values of $y_{\text {cut }}, b, \phi_{t}$, and $\epsilon$. These large uncertainties prevent a precise theoretical determination of the SFR in a cloud. Nevertheless, it is instructive to compare our determinations with observational ones. Such a comparison has partly been done in $\mathrm{HC} 11$ and will be extended here. The observed SFR per free-fall time in star-forming molecular clouds in the Milky Way lies in the range $0.03 \lesssim \mathrm{SFR}_{\mathrm{ff}} \lesssim$ 0.3 for clouds in the mass range $10^{3} \lesssim M_{c} / M_{\odot} \lesssim 10^{6}$, with $\mathcal{M} \approx 10-15$ and $\alpha_{\text {vir }} \approx 0.3-2$, with a mean value $\left\langle\mathrm{SFR}_{\mathrm{ff}}\right\rangle \approx 0.16$ (Murray 2011; Heyer et al. 2009). Looking at Galactic nearby molecular clouds and massive star-forming dense clumps, Heiderman et al. (2010) find SFRs in the range 

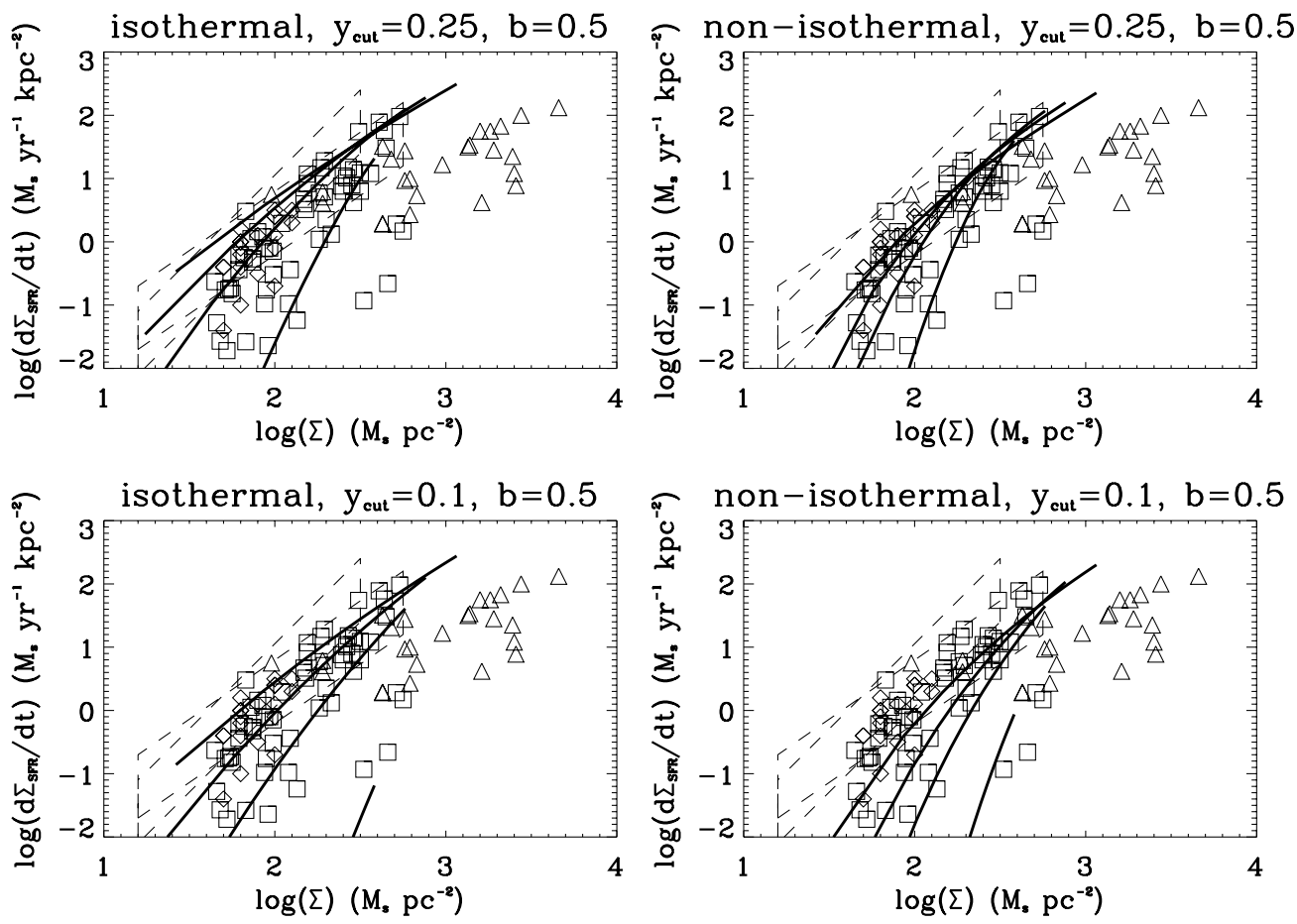

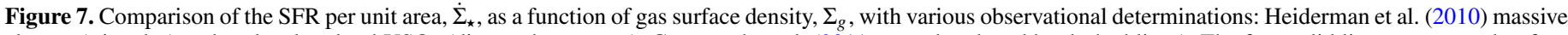

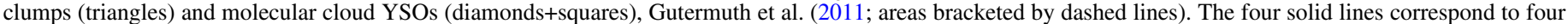
clump sizes, namely, $R_{c}=0.5,2,5$, and $20 \mathrm{pc}$ (from right to left).

$\approx 0.02-0.12$ for molecular clouds and $\approx 0.03-0.5$ for dense clumps, yielding a mean value $\approx 0.1$. These values are quite consistent with the ones derived above from our theory, while the typical rates obtained by KMK for similar conditions are about an order of magnitude smaller, as noted by Murray (2011) and Heiderman et al. (2010), a point we addressed in the previous section.

Traditionally, the observationally determined SFR for a given clump (or region of a clump) of mass $M_{c}$, radius $R_{c}$, lifetime $\tau_{0}$, and mean dynamical time $\tau_{\mathrm{ff}}^{0}$ is the projected SFR, i.e., the $S F R$ per unit area:

$$
\dot{\Sigma}_{\star}=\frac{\Sigma_{\star}}{\tau_{0}}=\operatorname{SFE} \times\left(\frac{\Sigma_{g}}{\tau_{0}}\right)=\operatorname{SFR}_{\mathrm{ff}} \times\left(\frac{\Sigma_{g}}{\tau_{\mathrm{ff}}^{0}}\right),
$$

where $\Sigma_{g}=M_{c} / \pi R_{c}^{2}=(4 / 3) \bar{\rho} R_{c}$ for a spherical clump is the clump gas surface density. The relationship between this projected SFR and the gas surface density is generally found to obey, within a large scatter, a power-law relationship:

$$
\dot{\Sigma}_{\star}=\Sigma_{100}\left(\frac{\Sigma_{g}}{100 M_{\odot} \mathrm{pc}^{-2}}\right)^{N} M_{\odot} \mathrm{yr}^{-1} \mathrm{kpc}^{-2} .
$$

Extragalactic studies yield the well-known Kennicutt-Schmidt relation, with $\Sigma_{100}=0.16 \pm 0.6$ and $N=1.4 \pm 0.15$.

Figure 7 displays $\dot{\Sigma}_{\star}$ predicted by Equation (42), as a function of surface density, $\Sigma_{g}$, for four typical clump sizes, namely, $R_{c}=$ $0.5,2,5$, and $20 \mathrm{pc}$, both in the isothermal and non-isothermal case. The impact of the uncertainties due to $y_{\text {cut }}$ is shown by the two calculations with $y_{\text {cut }}=0.1$ and 0.25 , respectively. The clumps are assumed to follow Larson's (1981) relations and thus have velocities and densities given by Equations (35) and (36), with $u_{0}=1$ and $d_{0}=0.1-5$, i.e., $\bar{n} \approx 10-10^{4} \mathrm{~cm}^{-3}$. The corresponding global and local Mach numbers entering our theory, $\mathcal{M}$ and $\mathcal{M}_{\star}$, are consistently derived from these values.
We have taken $\epsilon / \phi_{t}=0.1$ in the figure. Also displayed in the figure are the data of Heiderman et al. (2010), both for the class I and flat spectral energy distribution young stellar objects (YSOs) and the massive clumps, as well as the location of the observations of Gutermuth et al. (2011), as bracketed by the two large diamond areas. These latter authors determined the SFR in eight nearby molecular clouds, sampling gas surface density regions inferred from near-IR extinction mapping, ranging from $\sim 15$ to $300 M_{\odot} \mathrm{pc}^{-2}$. Their analysis is similar to that by Heiderman et al. (2010) but, as they examined larger clouds, they placed stronger statistical constraints on the SFR versus gas surface density determinations than that study. Moreover, these authors include pre-main-sequence Class II objects in addition to Class 0, I protostars, from the Spitzer and 2MASS surveys.

Several conclusions can be drawn from the theoryobservation comparison displayed in Figure 7.

1. All calculations reproduce well the observed values with the scatter, and exhibit a direct correlation between the density of star formation and the gas density, in agreement with the observational determinations.

Clearly, it seems difficult to provide a universal relation such as Equation (51) over the whole density range, given the strong dependence, both for the slope and the normalization, of the SFR upon the properties of the clump, i.e., its mass and the nature of turbulence forcing. What seems to be more robust is that at very high density, $\Sigma_{g} \gtrsim 300 M_{\odot} \mathrm{pc}^{-2}$, the theoretical relations seem to merge toward a strongly super-linear behavior with an exponent $N \simeq 2$ (an exact value is difficult to infer as it depends on the clump size) and a normalization $\Sigma_{300} \approx 32$. Star formation is thus predicted to increase basically quadratically with density, and thus to be largely dominated by the contribution of the densest (likely to be bound) clumps. Given the steeply decreasing number of clumps with increasing density, 
however, such dense clumps represent only a modest $(\lesssim 20 \%)$ mass fraction of the clouds (Kainulainen et al. 2011). This slope becomes progressively steeper as density decreases, the steeper the smaller the clump, as expected from the analysis conducted in the previous section.

This $N \simeq 2$ value for the slope is in excellent agreement with the values $N=1.67-2.67$ determined by Gutermuth et al. (2011) in eight nearby molecular clouds. Lower values, including Kennicutt-Schmidt like values, $N \sim 1.4$, with a star formation threshold are excluded by these authors.

2. The dispersion in the observed SFR determinations is well explained by variations among the clump sizes, not mentioning possible variations of the $\left(\epsilon / \phi_{t}\right)$ efficiency factor and of the contribution of different turbulence modes, as formalized by the factor $b$, between clumps.

3 . The theory, as the observations, predicts a severely decreasing SFR below a typical value $\Sigma_{g} \approx 100 M_{\odot} \mathrm{pc}^{-2}$, i.e., $N_{\mathrm{H}_{2}} \approx 5 \times 10^{21} \mathrm{~cm}^{-2}, \bar{n} \approx 2500(R / 1 \mathrm{pc})^{-1} \mathrm{~cm}^{-3}$, which corresponds to a visual extinction $A_{V} \approx 7$ (Draine 2003), except for the largest $(20 \mathrm{pc})$ clumps. We recall that such a drop in the SFR naturally arises from our theory, without invoking any density threshold.

4. There is no real density threshold in star formation. Indeed, according to our calculations, star formation is predicted to keep processing, although with a steadily decreasing rate, even at densities lower than the aforementioned value, but only at non negligible rates in the largest clumps. This was expected from the discussion in Section 3.2.2.1. In this low-density domain, our values can be compared with the observations of Gutermuth et al. (2011). As mentioned earlier, these authors examined larger clumps than previous studies and found larger SFR values than these latter at low density. The eight molecular cloud sample examined by these authors covers a range of clump sizes and surface densities $L_{c} \sim 4-20 \mathrm{pc}, \Sigma_{g} \sim 22-71 M_{\odot} \mathrm{pc}^{-2}$. In this range, they determine $\Sigma_{\star} / \Sigma_{g}^{2}$ in the range $\left(3 \times 10^{-4}\right)-(5 \times$ $\left.10^{-3}\right) \mathrm{pc}^{2} M_{\odot}^{-1}$. These values can be directly confronted with the ones inferred from the two upper solid lines, which correspond, respectively, to $R_{c}=5$ and $20 \mathrm{pc}$, in the appropriate surface density range. This yields $\Sigma_{\star} / \Sigma_{g}^{2} \approx$ $2.0 \times\left(10^{-5}-10^{-3}\right)$ for the non-isothermal case and values larger by about a factor 10 for the isothermal case, for our fiducial value of turbulence forcing, $b=0.5$. Remembering that both observational and theoretical determinations are subject to significant uncertainties, the agreement can be considered as satisfactory.

This reinforces our suggestion that the SFR smoothly decreases with decreasing gas density and that there is no real density threshold for star formation. The severe drop in the $\mathrm{SFR}$, which is predicted to occur around $\Sigma_{g} \approx 100 M_{\odot} \mathrm{pc}^{-2}$ for $\sim$ pc-size clumps, simply reflects the basic mechanisms mentioned in Section 3.2.2.1: star formation can still occur, although at significantly lower rates, in low-density clumps, provided that these latter, assuming they follow Larson's relation, are large enough to (1) significantly exceed their typical Jeans length and (2) generate enough large-scale turbulence, i.e., have large enough Mach numbers to generate a large enough spectrum of density fluctuations, and thus of prestellar cores. According to the observed clump size distribution, $d N_{c} / d L_{c} \propto L_{c}^{-1.9}$ (Kainulainen et al. 2011), however, such clumps are very rare.
This stresses the need to observe very large areas at low-density in order to get statistically significant SFR detections.

A threshold for core formation was also ruled out in the SCUBA survey of Perseus by Hatchell et al. (2005). These authors stress the existence of submillimeter cores, identified from IRAS as likely Class I objects, down to $A_{V}=3$, i.e., $N_{\mathrm{H}_{2}} \sim 10^{21} \mathrm{~cm}^{-2}$, and demonstrate the steeply decreasing probability of finding a core with decreasing column density. This is also consistent with the analysis of André et al. (2011, Figure 3), who find a drastically decreasing but non-zero number of Class 0 prestellar core candidates below about the aforementioned $A_{V} \sim 7$ value.

In contrast, several studies of various local clouds (Onishi et al. 1998; Johnstone et al. 2004; Enoch et al. 2007; Lada et al. 2010; André et al. 2010) do find a steep break in the SFR-gas density relationship around the aforementioned $\sim 100 M_{\odot} \mathrm{pc}^{-2}$ value that they identify as a density threshold for star formation. It should be stressed, however, that all these studies still find prestellar cores below this limit, suggesting that star formation continues, although with a much smaller efficiency, down to the limit of the surveys, i.e., $\Sigma_{g} \approx 10 M_{\odot} \mathrm{pc}^{-2}$. As mentioned above, a noticeable exception is the recent work by Gutermuth et al. (2011), who explore larger areas and who include pre-mainsequence Class II objects in addition to Class 0, I protostars, from the Spitzer and 2MASS surveys, and who do not find evidence for a column density threshold for star formation from 15 to $300 M_{\odot} \mathrm{pc}^{-2}$. These authors explain the different result by the very small number of Class 0 , I protostars at lowgas column densities, and thus the low statistics in previous surveys, including the Spitzer-derived data of Heiderman et al. (2010). Interestingly, by comparing the SFR derived from YSO surface densities and the ones derived from far-IR luminosity, as commonly used as a proxy of the SFR, they find that the $L_{\mathrm{FIR}^{-}}$ derived SFRs are systematically about an order of magnitude below the YSO-derived ones. This is confirmed, for instance, by the recent analysis of W40 and Serpens South with Herschel by Maury et al. (2011), who have access to Class 0 objects and find SFRs an order of magnitude higher than the typical SFRs observed for embedded infrared clusters. This suggests, if anything, that SFR determinations based only on Class 0 or Class I objects underestimate the real SFR by a fair amount. Therefore, the aforementioned identification of a cutoff in the SFR below $\sim 100 M_{\odot} \mathrm{pc}^{-2}$ might in fact be due to the very small number of Class 0 , I protostars at low-gas column densities and the too limited statistics (too small fields of view) in the surveys. This is supported by the recent analysis of Bressert et al. (2010) who show that using only near-infrared detections and small fields of view allows identifications of young stars only in the densest parts of the clouds, a bias that can be corrected with Spitzer data and larger surveys.

\subsection{Molecular Cloud versus Galactic SFR}

As already found in the observations of Heiderman et al. (2010) and Evans et al. (2009), our SFR-gas relation, characteristic of Galaxy star-forming molecular clouds, lies above the Kennicutt-Schmidt relation by about a factor of 10 at high density. It should be remembered, however, that the Kennicutt-Schmidt relation derives from extragalactic determinations and is averaged over much larger regions ( $\sim$ kpc-size) than individual molecular cloud complexes $(\lesssim 10-100 \mathrm{pc})$. Such an average determination includes starforming regions but also diffuse molecular gas or atomic gas that is not forming stars, leading to an overestimate of the 
amount of gas counted as star-forming gas (Heiderman et al. 2010). Recent SFR determinations in nearby galaxies indeed show that the measurement size scale used significantly changes the SFR-gas surface densities relation (Liu et al. 2011). Moreover, SFR in GMCs are determined from inventorying the YSOs and assuming a star formation timescale of $\sim$ Myr while galaxyaveraged SFRs are derived from conversion of an FIR flux into a mass growth rate assuming a timescale of 10-100 Myr, naturally leading to a smaller SFR for a given average gas density. Since, as shown in the present study, the SFR depends on the clump's mean density and significantly decreases for low-density clumps, this provides a plausible explanation for the difference between GMCs and extragalactic SFR-gas quantitative determinations. In fact, a correct determination of the SFR-gas relation at extragalactic scales should take into account the density dependence of the SFR-gas relation. The same explanation applies to the characteristic value of the SFR inferred for the Milky Way, found to be about an order of magnitude smaller than in GMCs. A likely explanation is that most of the mass of the clouds that compose the Galaxy is at low column density, with only a very small fraction in condensed structures at high enough density for efficient star formation (see, e.g., Kainulainen et al. 2011). Indeed, large-scale clouds which compose the Galaxy have densities around $\sim 10^{21} \mathrm{~cm}^{-2}$ $\left(\bar{n} \lesssim 100 \mathrm{~cm}^{-3}\right)$. Therefore, most of the clouds in the Milky Way no longer produce stars. On the other hand, our calculations suggest that the power dependence of the SFR upon the gas surface density in the (high-density) efficient star formation regime is not drastically different for Galactic and extragalactic determinations, since our exponent at high density is $N \approx 2$, whereas the one characteristic of the Kennicutt-Schmidt relation is $N=1.4 \pm 0.15$ (see Figure 7). Again, such a universal SFR-gas dependence naturally emerges from a picture of star formation being initiated by turbulence-induced fluctuations and the star formation rate being determined by the free-fall time of these fluctuations - not of the clump !- once gravity takes over, leading to a strong dependence of the SFR upon the gas density.

\subsection{Other Dependences of the Star Formation Rate}

In the previous section, only the SFR dependence upon the column density was examined. In the present section, we consider the SFR dependence upon the clump's mass and upon the column and volume densities over free-fall time. The results are compared with the sample of Heiderman et al. (2010).

\subsubsection{Star Formation Rate versus Clump Mass}

Figure 8 portrays the SFR, $\dot{M}_{\star}$, as a function of the cloud mass, $M_{c}$, for the isothermal and non-isothermal cases. Each line corresponds to the same clump sizes as in the previous figures, i.e., $R_{c}=0.5,2,5$, and $20 \mathrm{pc}$, with the normalization density $d_{0}$ increasing along the line from $d_{0}=0.1$ to 4.5 . We note the large scatter in the predicted $\dot{M}_{\star}$ for a given clump mass, in particular, for the non-isothermal case. The data of Heiderman et al. (2010) are displayed in the figure for comparison. The theoretical determinations agree very well with the data, indicating that within our theory, the range of clump characteristics we have investigated reproduces well the observationally determined SFR values with the observed scatter. The dashed line in the figure displays the relation derived by Lada et al. (2012), $\dot{M}_{\star}=\left(4.6 \times 10^{-8}\right)\left(M_{c} / M_{\odot}\right) M_{\odot} \mathrm{yr}^{-1}$. The present calculations show that this relation, although broadly correct, may not be an accurate representation of the real star formation process, as this
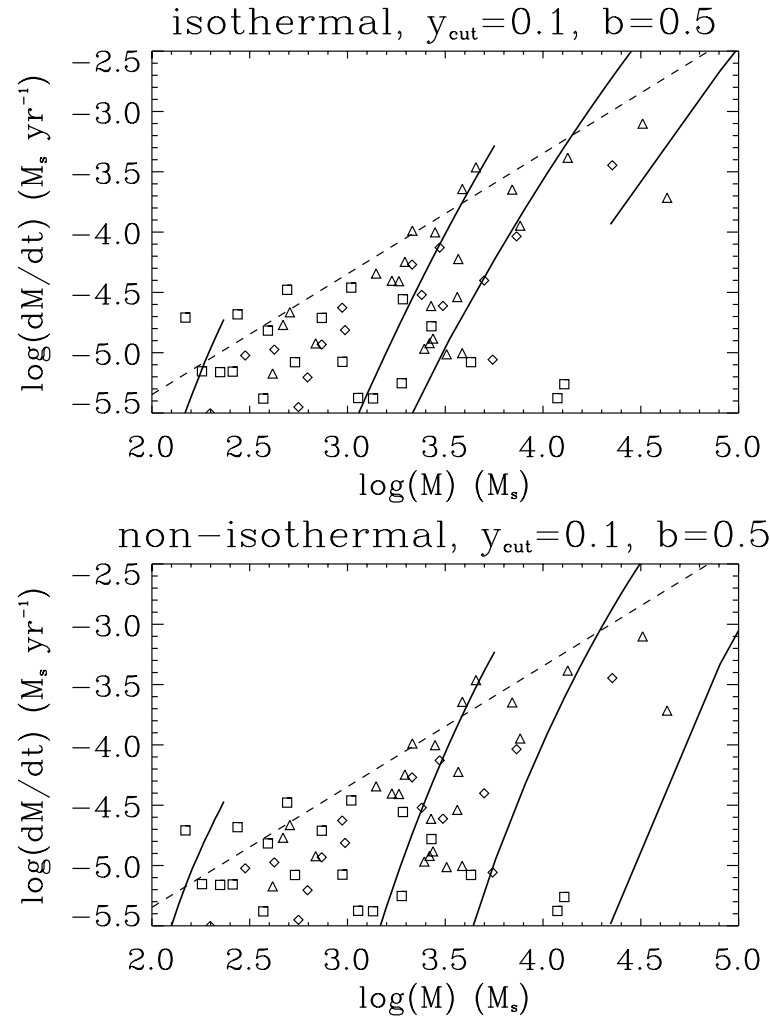

Figure 8. Star formation rate, $\dot{M}_{\star}$, as a function of the cloud mass, $M_{c}$, in the isothermal and non-isothermal cases. Each line corresponds to clump sizes $R_{c}=0.5,2,5$, and $20 \mathrm{pc}$, from left to right, with the normalization density $d_{0}$ increasing along the line from $d_{0}=0.1$ to 4.5 . The squares, triangles, and diamonds represent the data from Heiderman et al. (2010). The dashed line displays the fit of Lada et al. (2012).

latter not only depends much more steeply on the clump's mass, but also strongly depends on the clump's size.

\subsubsection{Dependence of Star Formation Rate on Free-fall Time}

The values of SFRs per mean free-fall time for a sample of clumps can be directly inferred by determining the slope of the relation $\dot{\Sigma}_{\star}$ versus $\left(\Sigma_{g} / \tau_{\mathrm{ff}}^{0}\right)$ (Equation (50)) or, equivalently, of the volume density relation $\dot{n}_{\star}$ versus $\left(n_{g} / \tau_{\mathrm{ff}}^{0}\right)$. A universal value of the SFR would correspond to one single, constant value of the slope for the whole sample.

To confront such observational determinations with our theory, we first vary the clump's size for a fixed density normalization parameter $d_{0}$. Indeed, assuming Larson's relation for our clumps, i.e., $\rho \propto R_{c}^{-0.7}$, yields $\Sigma \propto R_{c}^{0.3}$ and $\tau_{\mathrm{ff}} \propto$ $R_{c}^{0.35}$ for the column density and free-fall time, respectively. Therefore the column density over free-fall time, $\Sigma / \tau_{\mathrm{ff}}$, is almost independent of the clump's size. The corresponding $\dot{\Sigma}_{\star}$ versus $\left(\Sigma_{g} / \tau_{\mathrm{ff}}^{0}\right)$ curves are thus almost parallel to the $y$-axis, making the dependence upon $d_{0}$ and $R_{c}$ straightforward. Seven values of $d_{0}$ are computed, namely, $0.5,1,2,3,4,5$, and 10 as well as eleven sizes $R_{c}=0.5,1,2,4,6,8,10,12,14,16$, and 18 pc. Figure 9 displays the theoretical predictions for $\dot{\Sigma}_{\star}$ versus $\left(\Sigma_{g} / \tau_{\mathrm{ff}}^{0}\right)$ as well as the observational determinations of Heiderman et al. (2010). As is obvious in the figure, and as expected from the previous subsection, the overall agreement between our predictions and the data is very good and the observational scatter is well reproduced by the explored variations of clump sizes and densities. Note that given the large spread in the data 

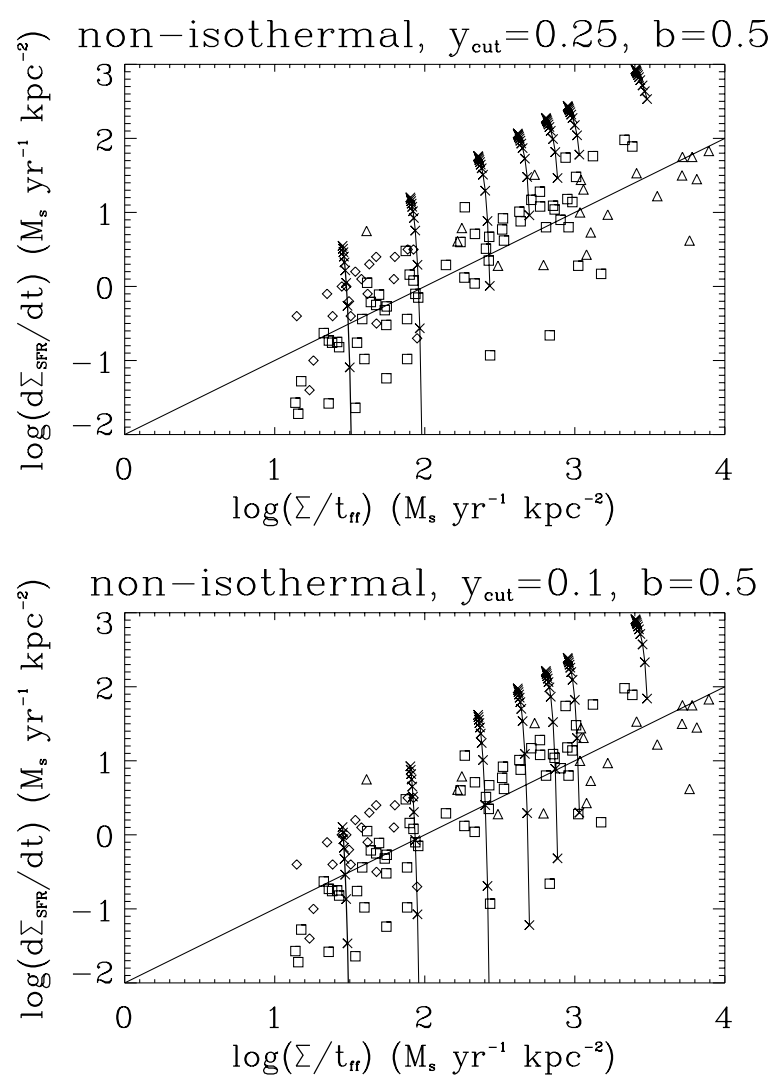

Figure 9. SFR per unit area, $\dot{\Sigma}_{\star}$, as a function of $\left(\Sigma_{g} / \tau_{\mathrm{ff}}^{0}\right)$ for 7 values of $d_{0}=$ $0.5,1,2,3,4,5$, and 10 (left to right) and 11 clump sizes $R_{c}=0.5,1,2,4,6,8$, $10,12,14,16$, and $18 \mathrm{pc}$ (from bottom to top on each line, as indicated by the crosses). Each curve corresponds to a constant $d_{0}$. The highest values of $d_{0}$ and $R_{C}$ correspond to the highest SFRs.

and the uncertainties discussed previously in the theory, it is difficult to assess more than a qualitative agreement.

Interestingly, the confrontation between our results and the observed SFR values from Figure 9 leads to the conclusion that the size of star-forming clumps should decrease with increasing column density over free-fall time. Typically, the clump's size should be less than about 2 pc for $\log \left(\Sigma / \tau_{\mathrm{ff}}\right) \simeq 3$ and less than about $0.5 \mathrm{pc}$ for $\log \left(\Sigma / \tau_{\mathrm{ff}}\right) \simeq 3.5$ (with a dispersion of a factor 2-3). To verify whether this trend is indeed real, we have plotted $\dot{\Sigma}_{*}$ as a function of the clump's radius (Figure 10). Indeed, Figure 10 shows that a clear trend for the highest values of $\dot{\Sigma}_{\star}$ has been obtained for the smallest clouds. The reason of this behavior is not completely clear but appears to be an important element to understanding the SFR dependence. A likely explanation is that large clouds never become dense enough, i.e., massive enough (see, e.g., Figure 8). More precisely, as massive clouds collapse, they undergo local gravitational fragmentation in dense clumps where most of the star formation occurs.

Finally, for completeness we also display $\dot{n}_{\star}$ versus $\left(n_{g} / \tau_{\mathrm{ff}}^{0}\right)$ in Figure 11 for the same cloud parameters as in Figure 9 (the various curves correspond to a fixed $d_{0}$ ), while Figure 12 shows the results for the same cloud parameters as in Figure 7. For the data of Heiderman et al. (2010), we derived the volume density from the surveyed area as (assuming spherical clumps of area A) $\bar{\rho}=(3 \sqrt{\pi} / 4)\left(M_{c} / A^{3 / 2}\right)$.

According to the present analysis, there is clearly no "universal" slope value either for the $\dot{\Sigma}_{\star}$ versus $\left(\Sigma_{g} / \tau_{\mathrm{ff}}^{0}\right)$ or for $\dot{n}_{\star}$ versus $\left(n_{g} / \tau_{\mathrm{ff}}^{0}\right)$ relation. Instead, these relations signifi-

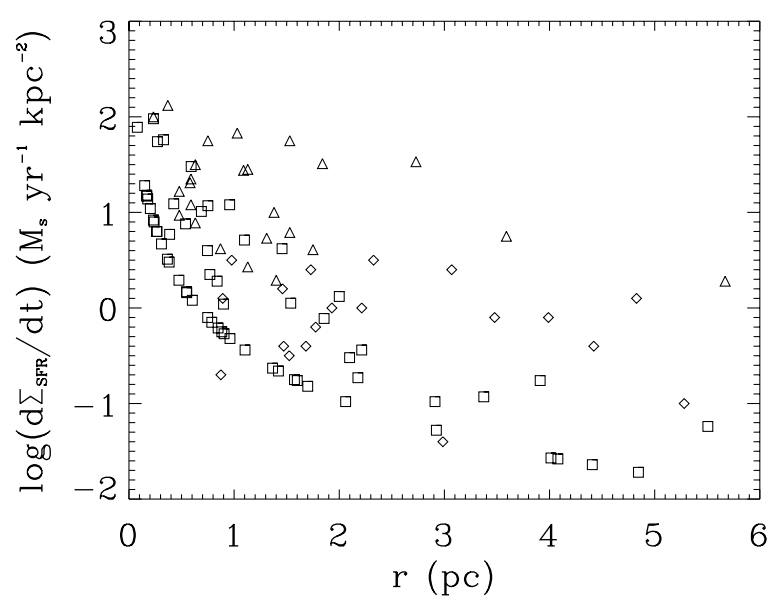

Figure 10. $\dot{\Sigma}_{\star}$ as a function of the clump radius, $r$. There is a clear trend of $\dot{\Sigma}_{\star}$ strongly increasing with decreasing clump's size, particularly if the massive clump determinations (triangles), which tend to differ from molecular cloud YSOs (diamonds and squares), are excluded.
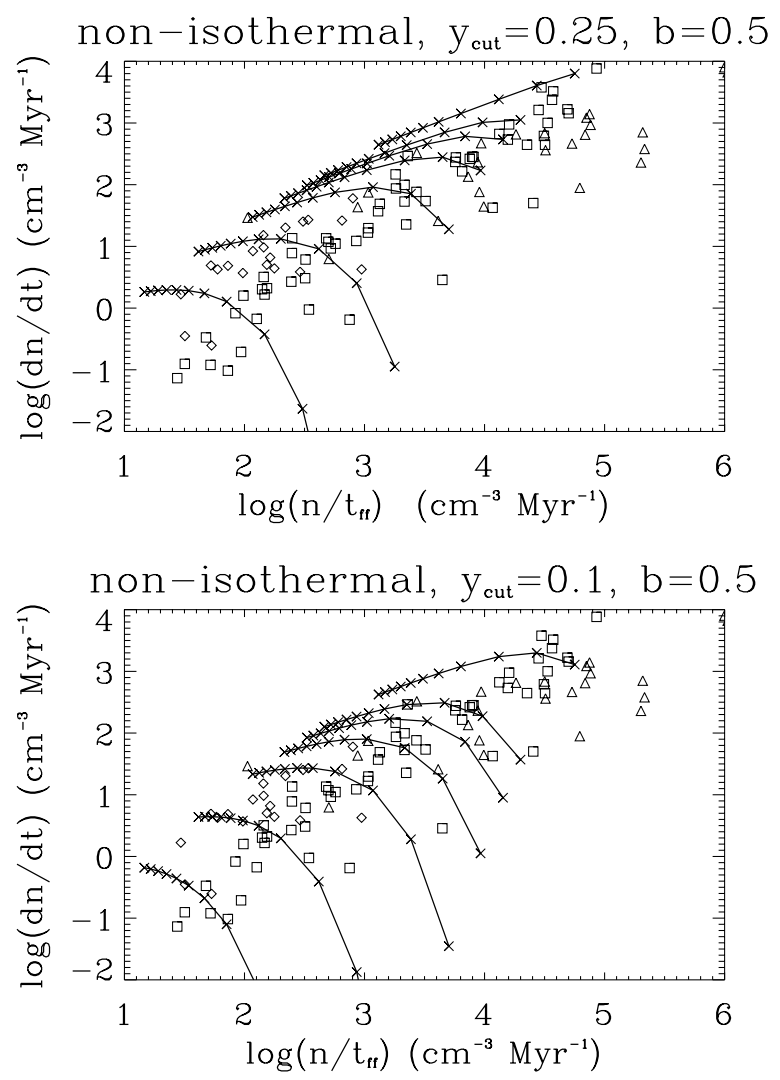

Figure 11. Volumic SFR density, $\dot{n}_{\star}$, as a function of $\left(n_{g} / \tau_{\mathrm{ff}}^{0}\right)$ for the same conditions as in Figure 9. Each line represents a fixed value of $d_{0}$.

cantly vary with the clump's size and density. Indeed, below $\Sigma / \tau_{\mathrm{ff}} \lesssim 1000 M_{\odot} \mathrm{yr}^{-1} \mathrm{kpc}^{-2}$, there is between 1 and 2 orders of magnitude variations in the SFR. Even in dense regions corresponding to $n / \tau_{\mathrm{ff}}>100 \mathrm{~cm}^{-3} \mathrm{Myr}^{-1}$, the mean value of the SFR, as measured by the slope of the illustrated relations, typically varies between $\sim 3 \%$ and $\gtrsim 10 \%$. This analysis is in contrast with the claim by Krumholz et al. (2012) for a universal SFR value of about $1 \%$ from clouds to starburst galaxies. As mentioned earlier, molecular clouds are very clumpy structures with orders of magnitude of density variations. Therefore, it seems difficult to invoke an average timescale, defined at the cloud's mean density, as the relevant timescale for star formation. 

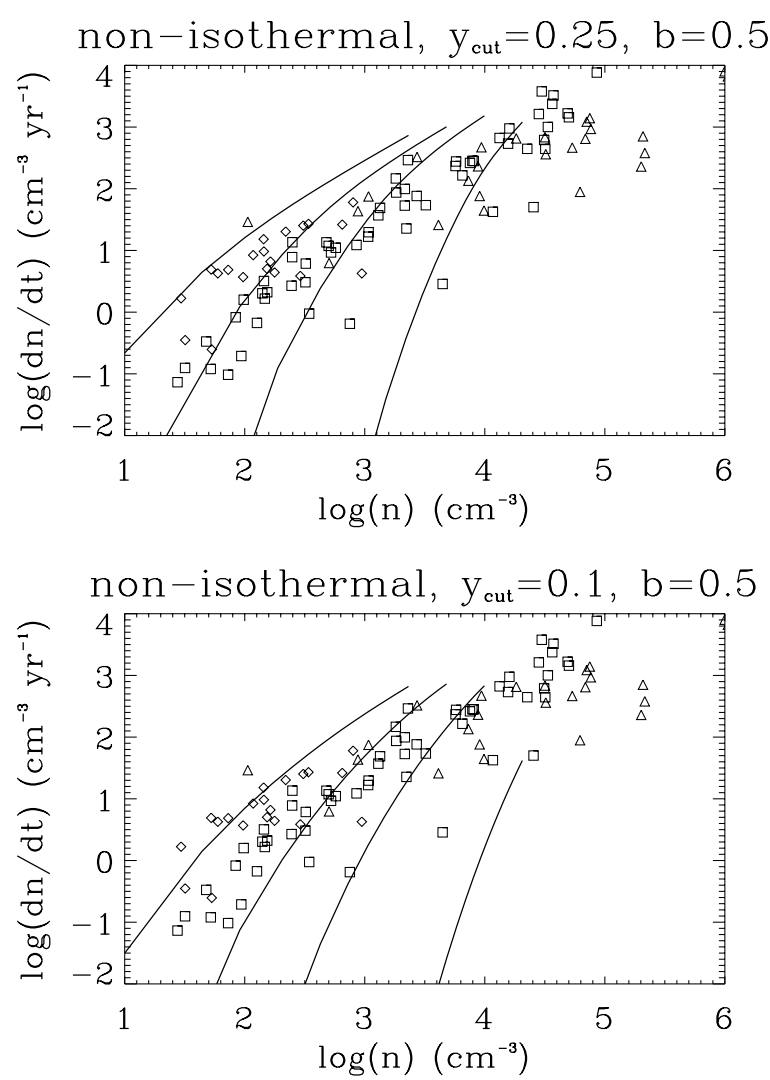

Figure 12. Volumic SFR density, $\dot{n}_{\star}$, as a function of $\left(n_{g} / \tau_{\mathrm{ff}}^{0}\right)$ for the same conditions as in Figure 7. The four solid lines correspond to four clump sizes, namely, $R_{c}=0.5,2,5$, and 20 pc (from right to left).

\subsection{Discussion: The Case of Filaments}

According to various recent studies, in particular the ones conducted with Herschel, star formation is found to occur in filaments (André et al. 2010; Men'shchikov et al. 2010; WardThompson et al. 2010; Molinari et al. 2010; Hill et al. 2011). It is well known that (unbound or bound) molecular clouds exhibit a filamentary structure, as expected from both hydrodynamical and MHD compressible (shock dominated) turbulence, even in the absence of gravity (Padoan et al. 2001; Nakamura \& Li 2008; Federrath et al. 2010; see also Banerjee et al. 2009). It is thus not surprising that the locations of star-forming populations tend to follow this morphology. We recall that in our formalism, the prestellar cores, progenitors of the protostars identified as Class 0 objects, are born in turbulence-induced clumps of enhanced density and isolate themselves from the surrounding medium under the action of gravity (Hennebelle \& Chabrier 2008; Chabrier \& Hennebelle 2011). For the sake of simplicity, the clumps and the cores in our theory are assumed to have a spherical geometry. Nonlinear simulations of the collapse of filaments show that the resulting fragments are nearly spherical (Inutsuka \& Miyama 1997). It thus seems reasonable to assume spherical collapse for the cores. The reality might be more complex for the star-forming clumps, which may have a flattened, filament-like geometry, with some of these filamentary clumps becoming themselves gravitationally unstable as they both accrete mass and dissipate turbulent energy, eventually yielding global collapse of the clump. Interestingly, the very same Herschel observations reveal that protostellar cores seem to be present only in the gravitationally bound clumps (André et al. 2010; Molinari et al. 2010; Arzoumanian et al. 2011), suggesting that star formation takes place dominantly in such clumps, prone to gravitational fragmentation. Gravitational contraction will thus cause filamentary clumps to fragment along the axis.

This possible peculiar role of filaments in star formation might affect our results by numerical factors of the order of a few but it does not fundamentally modify our theory of clump and prestellar core formation, and of the resulting CMF. ${ }^{11}$ In our theory, the (scale-free) criterion for clump formation is simply set up by an arbitrary critical density threshold $\delta_{c}=\log \left(\rho_{c} / \bar{\rho}\right)$ (see Section 3 of Paper I). The argument above thus suggests to choose for this threshold condition for the star-forming clumps-identified as the aforementioned filaments - the density at which these latter become gravitationally unstable and thus exceed a critical mass per unit length $M_{\text {crit }}=2\langle\sigma\rangle^{2} / G$, where $\langle\sigma\rangle$ includes both the thermal and non-thermal velocity dispersion contributions (Ostriker 1964; Larson 1985; Inutsuka \& Miyama 1992; Fiege $\&$ Pudritz 2000). For a temperature $T \sim 10 \mathrm{~K}$, this corresponds to a mean column density $N_{\mathrm{H}_{2}} \simeq 10^{22} \mathrm{~cm}^{-2}$, similar to the value identified in Section 4.1 for efficient star formation (point (3)), about 5-10 times the typical mean density of the surrounding cloud. This particular choice for the clump formation in our theory will not affect the resulting CMF determined by the virial condition for spherical collapse, as mentioned above.

Furthermore, a closer inspection of Table 1 and Figure 8 of Paper II (see also Figure 2) shows that small-size, low-density $\left(\lesssim 3000 \mathrm{~cm}^{-3}\right)$ clumps for which the CMF is very narrow and thus star formation is negligible, are unbound or only marginally bound $\left(\left(E_{\text {kin }}+E_{\text {th }}\right) \gtrsim\left|E_{\text {grav }}\right|\right)$, whereas the ones above about this density are bound and thus prone to collapse. Therefore, our theory suggests that the clumps in which star formation is taking place dominantly should be denser than $\bar{n} \sim$ a few $10^{3} \mathrm{~cm}^{-3}$, i.e., $N_{\mathrm{H}_{2}} \simeq 10^{22} \mathrm{~cm}^{-2}$ for pc-size clumps, in fairly good agreement with the aforementioned critical value inferred for filaments. This is consistent with the results found in Section 3.2.2.2, which show that the SFR is highest in the largest and densest clumps, with $\alpha_{\text {vir }} \lesssim 1$. Note that given the scale-free nature of the turbulent flow responsible for the clump formation, such a minimum density for efficient star formation must apply at all scales, and thus must vary accordingly with the clump's size. The aforementioned value typically applies for a $1 \mathrm{pc}$ size clump and should increase as $\sim R_{c}^{-0.7}$ for smaller sizes, providing Larson's relations apply.

Therefore, a picture where star formation occurs dominantly in relatively dense, rather bound clumps with mean density $\bar{n} \gtrsim 3000 \mathrm{~cm}^{-3}$, in agreement with observational results, naturally emerges from our theory. The filamentary nature of the clumps does not significantly affect the results, except for possible numerical factors of the order of unity.

\section{INFLUENCE OF THE CLUMP DISTRIBUTION AND EVOLUTION}

So far, we have considered the core mass spectrum produced within a single clump with fixed physical quantities (mass, size, density). In real situations, there will be a distribution of evolving clumps. In this section, we investigate the impact on the gravitationally bound core mass spectrum of the time dependence of the cloud parameters as well as having a clump distribution rather than a single clump. In order to distinguish the influence of these two effects, we first consider the time

\footnotetext{
11 The characteristic Jeans mass for a nearly isothermal filament $(\gamma \sim 1)$
} differs only by a factor 0.6 from the one for a sphere (Larson 2003). 
evolution of a single clump before investigating the effect of a distribution.

\subsection{A Simple Model of Clump Evolution}

One of the intrinsic difficulties in inferring the mass spectrum of the self-gravitating fluctuations that may eventually lead to stars is the choice of clump parameters (density, size, velocity dispersion, etc.). As seen from our calculations, these clump properties have a strong impact on the mass spectrum. The peak position, for instance, is determined by the clumps Jeans mass, and thus mean density, temperature, and Mach number. Clumps, however, are not well-defined entities, in particular because they constantly grow in mass by accretion, so that their density and size evolve with time. Under these conditions, it is clear that the choice of a fixed set of clump parameters is a simplification. A proper investigation of the clump evolution would require us to develop a fully self-consistent model for clump formation and evolution, including growth by accretion, gravitational contraction, or turbulence dispersion as well as spatial inhomogeneity. There is no such model of clump evolution yet; we thus consider a very simple prescription, essentially inspired by observations.

As mentioned above, instead of setting a density and size, we assume that the clump is accreting at a fixed accretion rate, $\dot{M}$. Starting with a mass $M(t=0) \equiv M_{0}$, the clump mass grows as $M_{c}=M_{0}+\dot{M} t$. This mass entails the mass of the gas, $M_{g}$, and the mass of the stars, $M_{*}$, that formed in the clump, $M_{*}=M_{c}-M_{g}$. We further assume that the clump follows Larson-type relations as stated by Equations (35) with $d_{0}=3$ and $u_{0}=1$, which correspond to the canonical values of these relations. Then, knowing $M_{g}(t)$, we can infer the gas mean density, $\overline{n_{g}}(t)$, and thus the cloud radius $R_{c}(t)$. Since at a given time, $t$, the clump's parameters are well determined, we can calculate the SFR from our CMF/IMF theory, as stated by Equations (42) and (43). We can thus estimate the gas mass fraction converted into stars between $t$ and $t+d t$ as $d M_{*}(t)=M_{g} \times \operatorname{SFR}_{\mathrm{ff}} \times d t$. The total mass of stars at time $t, M_{*}(t)$, is thus

$$
M_{*}(t)=\int_{0}^{t} M_{g}(t) \operatorname{SFR}_{\mathrm{ff}}(t) d t .
$$

We typically integrate from 0 to $10 \mathrm{Myr}$ with time steps $d t=10^{5}$ yr. This procedure presents similarities with the model presented in Zamora-Avilés et al. (2012).

The accretion rate onto the molecular clumps is a difficult quantity to infer observationally and thus is not well known. Using observations of the LMC, Kawamura et al. (2009) and Fukui et al. (2009) propose that GMCs with a mass of $\simeq \mathrm{a}$ few $10^{5} M_{\odot}$ are accreting at a rate $\dot{M}=(1-5) \times 10^{-2} M_{\odot} \mathrm{yr}^{-1}$. To estimate the accretion rate on smaller clumps, we simply use the Larson relations,

$$
\begin{aligned}
\dot{M} \simeq & \frac{M}{\tau_{c}} \simeq \frac{M}{R_{c} /(\sigma / \sqrt{3})} \\
\simeq & M^{\frac{1.3+\eta}{2.3}} \frac{u_{0}}{\sqrt{3}}\left(\frac{4 \pi}{3} d_{0}\right)^{\frac{1-\eta}{2.3}}(1 \mathrm{pc})^{\frac{0.7-3 \eta}{2.3}} \\
\simeq & 10^{-3}\left(\frac{M}{10^{4} M_{\odot}}\right)^{\frac{1.3+\eta}{2.3}}\left(\frac{\sigma}{0.8 \mathrm{~km} \mathrm{~s}^{-1}}\right) \\
& \times\left(\frac{\bar{n}}{1 \mathrm{~cm}^{-3}}\right)^{\frac{1-\eta}{2.3}} M_{\odot} \mathrm{yr}^{-1},
\end{aligned}
$$
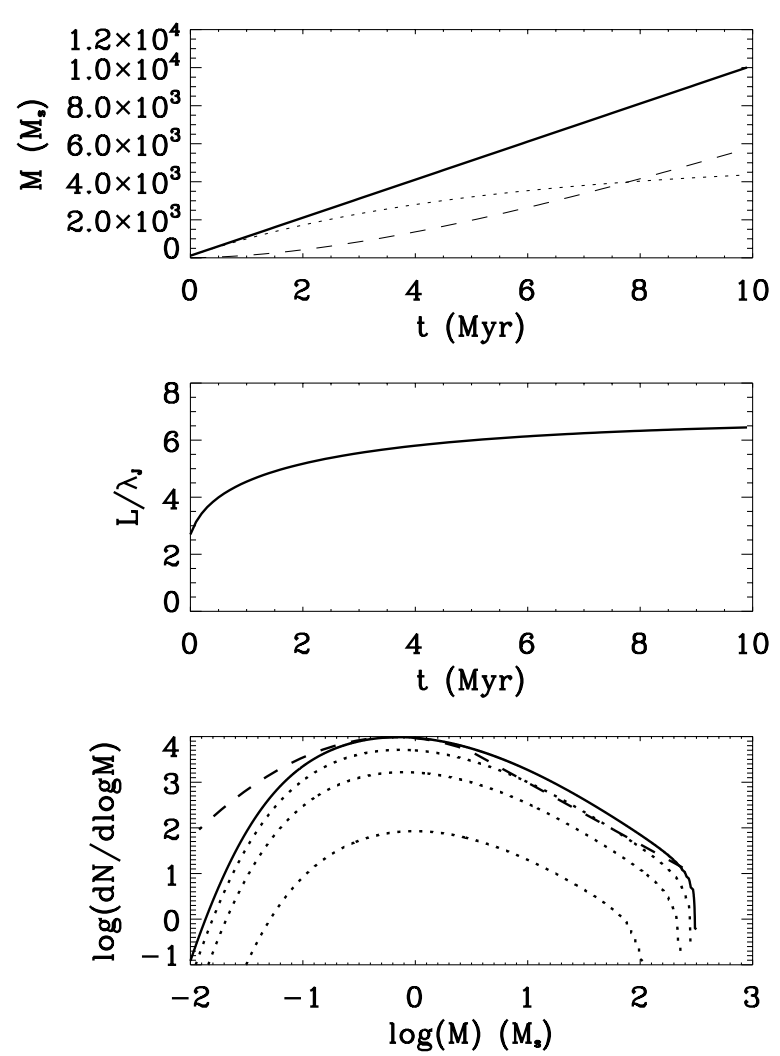

Figure 13. Clump evolution for $\dot{M}=10^{-3} M_{\odot} \mathrm{yr}^{-1}$ and $\epsilon / \phi_{t}=0.1$. Top panel: the total cloud mass (solid line), gas mass (dotted line), and star mass (dashed line). Middle panel: cloud radius over Jeans length. Bottom panel: mass spectrum after different times: $t=1,4$, and $7 \mathrm{Myr}$ (dotted lines from bottom to top) and $t=10 \mathrm{Myr}$ (solid line). The dashed line displays the SCIMF.

where the numerical constant has been estimated for $\eta=0.4$. For a GMC of mass $10^{5} M_{\odot}$, this estimate yields an accretion rate $\dot{M} \simeq 5 \times 10^{-3} M_{\odot} \mathrm{yr}^{-1}$, slightly lower but nevertheless close to the estimate of Fukui et al. (2009). For a cloud of $10^{3} M_{\odot}$, we get $2 \times 10^{-4} M_{\odot} \mathrm{yr}^{-1}$. We have thus explored three typical values for the accretion rate, namely, $\dot{M}=10^{-2}, 10^{-3}$, and $10^{-4} M_{\odot} \mathrm{yr}^{-1}$, which correspond to clumps of approximately $10^{5}, 10^{4}$, and $10^{3} M_{\odot}$.

Figure 13 portrays the results for $\dot{M}=10^{-3} M_{\odot} \mathrm{yr}^{-1}$ and $\epsilon / \phi_{t}=0.1$. The top panel shows the total mass of the clump (solid line), the mass of gas (dotted line), and the stellar mass (dashed line). While the total mass grows linearly with time, the mass of gas, which was initially almost equal to the total mass, increases less and less rapidly as star formation is occurring. At the beginning of the process, the mass of the cloud is small, and the amount of stars formed remains fairly limited. After $t \simeq 4 \mathrm{Myr}$, the stellar mass starts increasing significantly and after $t \simeq 8 \mathrm{Myr}$, the masses of gas and stars are roughly equal. After this time, the gas mass basically saturates, as the gas added to the cloud is rapidly converted into stars. Consequently, the size of the cloud (middle panel) increases rapidly at the beginning and much more slowly as star formation proceeds. The bottom panel displays the stellar mass spectrum at different times, namely, after 1, 4, and $7 \mathrm{Myr}$ (dotted lines from bottom to top) and after $t=10 \mathrm{Myr}$ (solid line); the dashed line corresponds to the SCIMF. As seen in the figure, the shape of the mass spectrum remains about the same over time but of course the integral, i.e., the total number of stars, strongly increases, a consequence of the growing size of the clump, as mentioned above. 

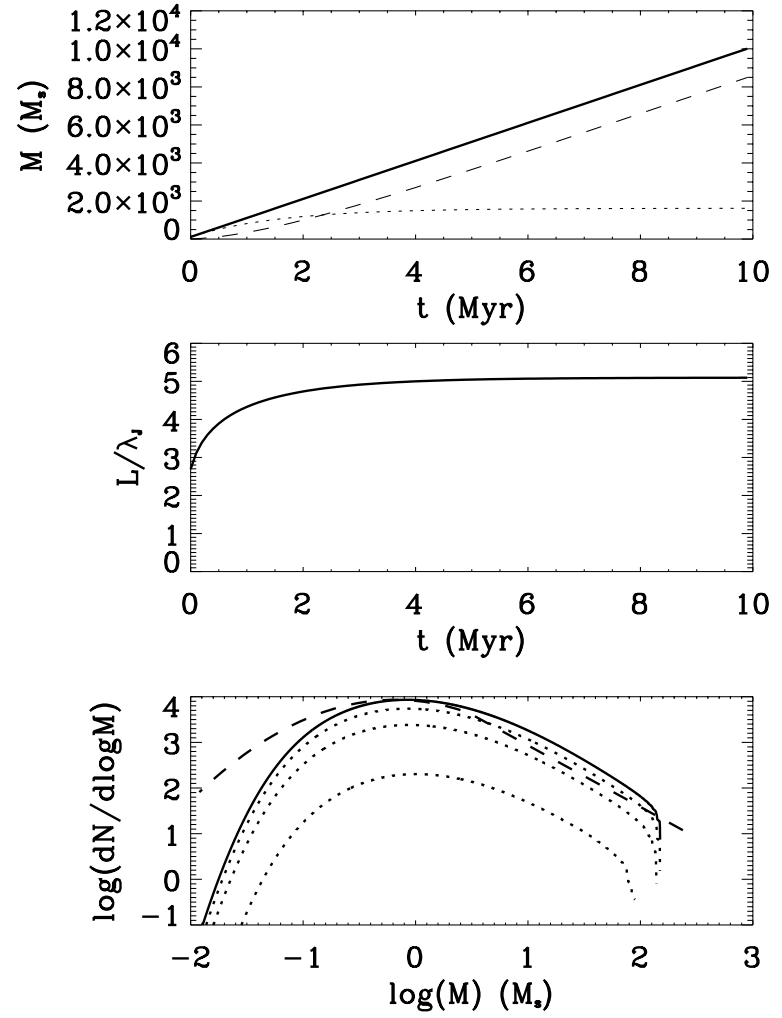

Figure 14. Same as Figure 13 for $\dot{M}=10^{-3} M_{\odot} \mathrm{yr}^{-1}$ and $\epsilon / \phi_{t}=0.3$.

Figure 14 displays similar results for the same accretion rate, $\dot{M}=10^{-3} M_{\odot} \mathrm{yr}^{-1}$, but $\epsilon / \phi_{t}=0.3$. The behavior remains qualitatively similar except that, as expected, stars form faster, since the SFR is three times larger (see Equation (43)), and thus the gas mass remains smaller by a factor of about 2 compared with the previous case. Consequently, the size of the clump is slightly smaller and less small-mass objects form because the Mach number $\left(\mathcal{M} \propto R_{c}^{\eta}\right)$ is slightly smaller (see Paper I).

Figure 15 displays the results for $\dot{M}=10^{-2} M_{\odot} \mathrm{yr}^{-1}$. The SFR is slightly smaller than for $\dot{M}=10^{-3} M_{\odot} \mathrm{yr}^{-1}$ because the clump grows more rapidly and thus is less dense. Interestingly, the mass spectrum remains very similar, except for the slightly larger number of low-mass objects, a consequence of the larger Mach number. Indeed, as discussed in Paper I (Equation (47) of Paper I), there is a partial compensation between the Jeans mass and the Mach number scale dependences as a function of the cloud's size/mass so that the location of the peak of the CMF remains almost unchanged. It is interesting to compare the top panel of this figure with the top panel of Figure 5 of VázquezSemadeni et al. (2007), which shows the total gas+stellar masses in a simulation of colliding flows. The general behavior and even some of the details of the simulation are very similar to the results portrayed in Figure 15: (1) the total mass of the cloud in both cases is a few $10^{4} M_{\odot} ;(2)$ the time at which stars and gas masses are equal agree within a factor of about 2 (particularly if we choose for the beginning of the cloud formation in the simulation the time $t \simeq 10 \mathrm{Myr}$, which seems more accurate than $t=0$; (3) when stars start forming efficiently, the mass of the gas remains nearly constant both in the simulation and in the present model. The main differences appear in the evolution of the stellar mass, which is more sudden in the simulations and faster at the beginning than in the later phases, in contrast to the present results. Moreover, while in the model the mass of
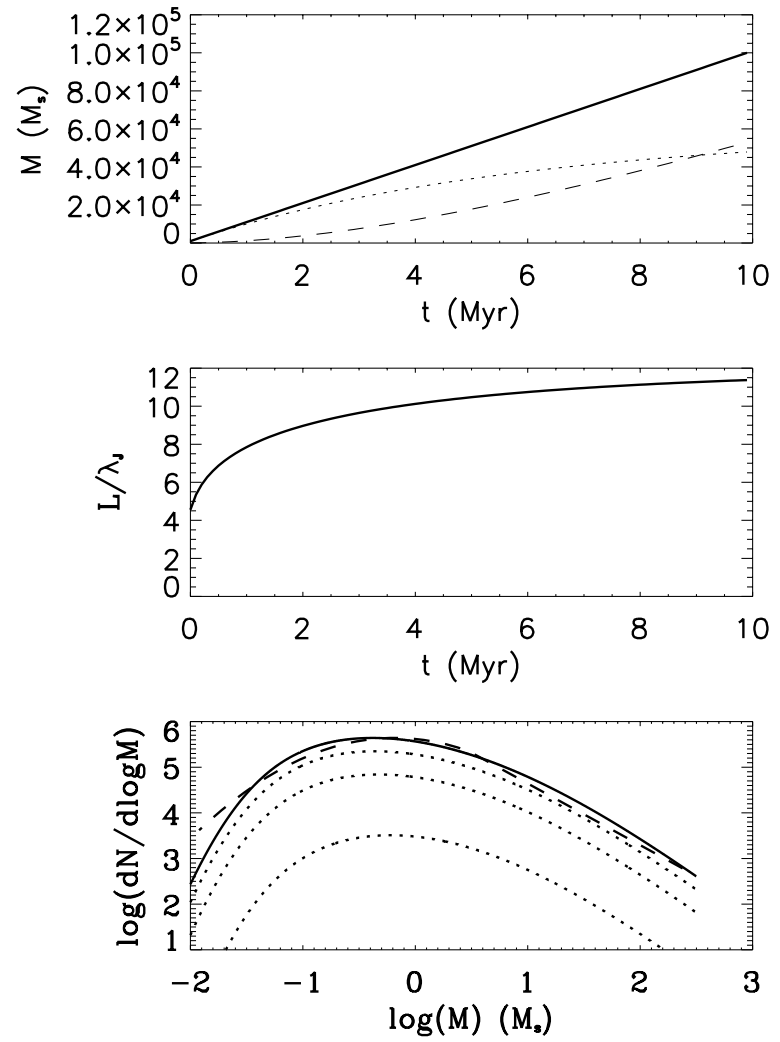

Figure 15. Same as Figure 13 for $\dot{M}=10^{-2} M_{\odot} \mathrm{yr}^{-1}$ and $\epsilon / \phi_{t}=0.1$.

the gas always increases, it decreases in the simulation. This is likely due to gravity, whose effects on the cloud dynamics are ignored in our model.

The last case we investigate is presented in Figure 16, which displays results for $\dot{M}=10^{-4} M_{\odot} \mathrm{yr}^{-1}$. The mass of the clump is only about $400 M_{\odot}$. The mass spectrum peaks at about the same mass for the reason mentioned above, but the mass spectrum is much narrower with a significant deficit of both massive and low-mass objects. This stems from the fact that for such small clumps, the Jeans length becomes comparable to the size of the cloud, as discussed earlier, drastically decreasing star formation (see, e.g., Figures 2 and 8 of Paper II).

\subsection{A Distribution of Time-dependent Star-forming Clumps}

It is now relatively well established that not only CO clumps (Heithausen et al. 1998; Kramer et al. 1998) but also infrared dark clouds (Peretto \& Fuller 2010) follow a mass distribution $\mathcal{N}^{\mathrm{cl}}=d N^{\mathrm{cl}} / d M \propto M^{-\gamma_{\mathrm{cl}}}$ with $\gamma_{\mathrm{cl}} \simeq 1.7$. It is important to recall that our theory predicts that this exponent, as well as the one for gravitationally bound cores when a gravitational collapse condition is taken into account, is a direct consequence of turbulence and is indeed related to the index $n^{\prime}$ of the power spectrum of $\log \rho$ by the relation $\gamma_{\mathrm{cl}}=1+x=3-n^{\prime} / 3$ (see Paper I). Therefore, the present theory naturally takes into account the clumpy structure of the gas and in principle no further calculations are needed. In other words, for spatially close enough clumps surrounded by sufficiently dense gas, there is no need to sum up over a clump population. While strictly true as long as (1) the gas is barotropic and (2) the density PDF is lognormal, this is no longer the case in real situations. Indeed, the diffuse ISM does not follow our Equation (31), as the atomic hydrogen that fills up the Galaxy can be as warm as 

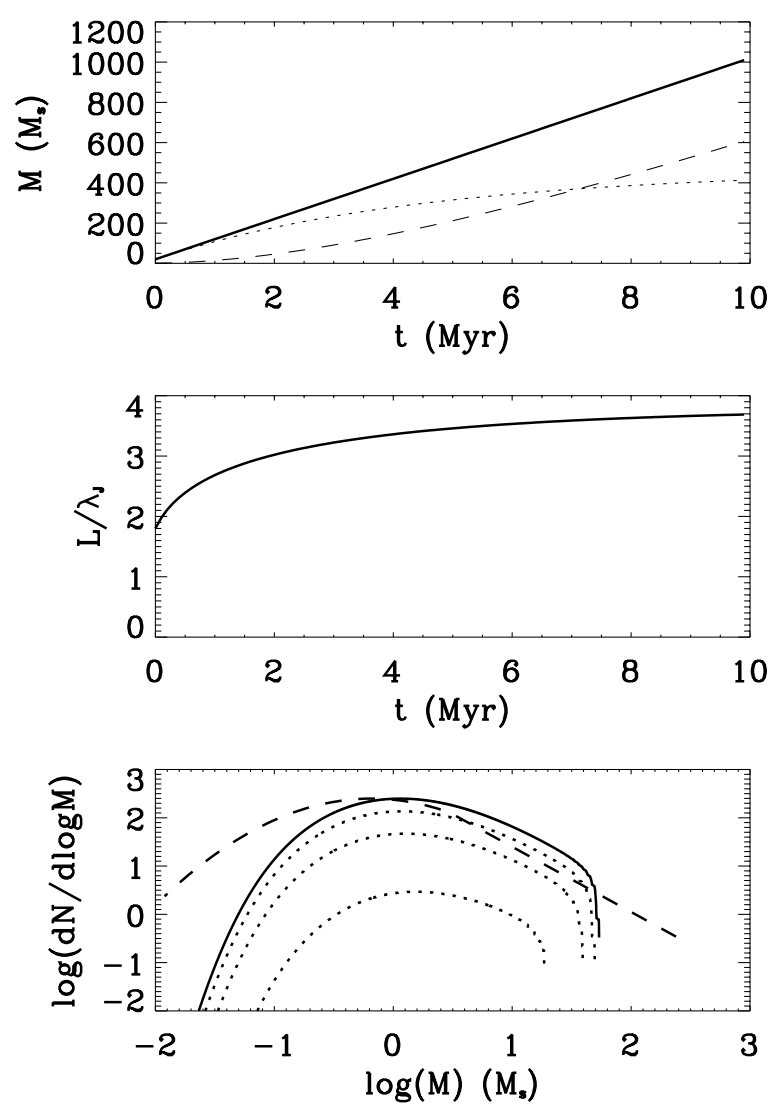

Figure 16. Same as Figure 13 for $\dot{M}=10^{-4} M_{\odot} \mathrm{yr}^{-1}$.

$10^{4} \mathrm{~K}$. Therefore, for clumps that are spatially well separated, the in-between gas is not isothermal and we must sum up over a clump population. The scale at which this happens is not well determined. Numerical simulations by Vázquez-Semadeni et al. (2007), Hennebelle et al. (2008), Heitsch et al. (2008), and Banerjee et al. (2009) suggest that the warm gas is tightly mixed with the cold gas at scales of about a few parsecs.

We calculate the mass spectrum resulting from the distribution of the time-dependent clumps described in the previous section. For that purpose, we proceed as follows. We perform a series of time-dependent clump calculations, choosing values of $\dot{M}$ between $10^{-1}$ and $10^{-5} M_{\odot} \mathrm{yr}^{-1}$ using logarithmic intervals. We stop the integration when $M_{*}=M_{g}$ in the clump and we adopt for the mass of the clump the value $M_{\mathrm{cl}}=M_{*}+M_{g}$. Then we sum up the corresponding mass spectra after multiplying by the aforementioned clump distribution $\mathcal{N}^{\mathrm{cl}}\left(M_{\mathrm{cl}}\right) \propto M^{-\gamma_{\mathrm{cl}}}$. The final core mass spectrum is given by

$$
\mathcal{N}_{\text {tot }}=\int_{0}^{M_{\text {sup }}} \mathcal{N}_{M_{c}}\left(M_{\mathrm{cl}}\right) V_{c l} \mathcal{N}^{\mathrm{cl}}\left(M_{\mathrm{cl}}\right) d M_{\mathrm{cl}},
$$

where $\mathcal{N}_{M_{c}}\left(M_{\mathrm{cl}}\right)=d n / d M_{c}$ is the mass spectrum of selfgravitating fluctuations (i.e., cores), i.e., the CMF, for a clump of mass $M_{\mathrm{cl}}$ and volume $V_{\mathrm{cl}}$, as given by Equation (2) (see Paper I and Paper II), and $M_{\text {sup }}$ is the largest mass in our sample which corresponds to the largest accretion rate.

At this stage, it is worth stressing that since $\gamma_{\mathrm{cl}}<2$, most of the mass is contained in the most massive clumps; $\gamma_{\mathrm{cl}}=2$ then corresponds to a critical case. We have thus considered three values of $\gamma_{\mathrm{cl}}$, namely $1.5,1.7$, and 2 . Figure 17 displays the results.
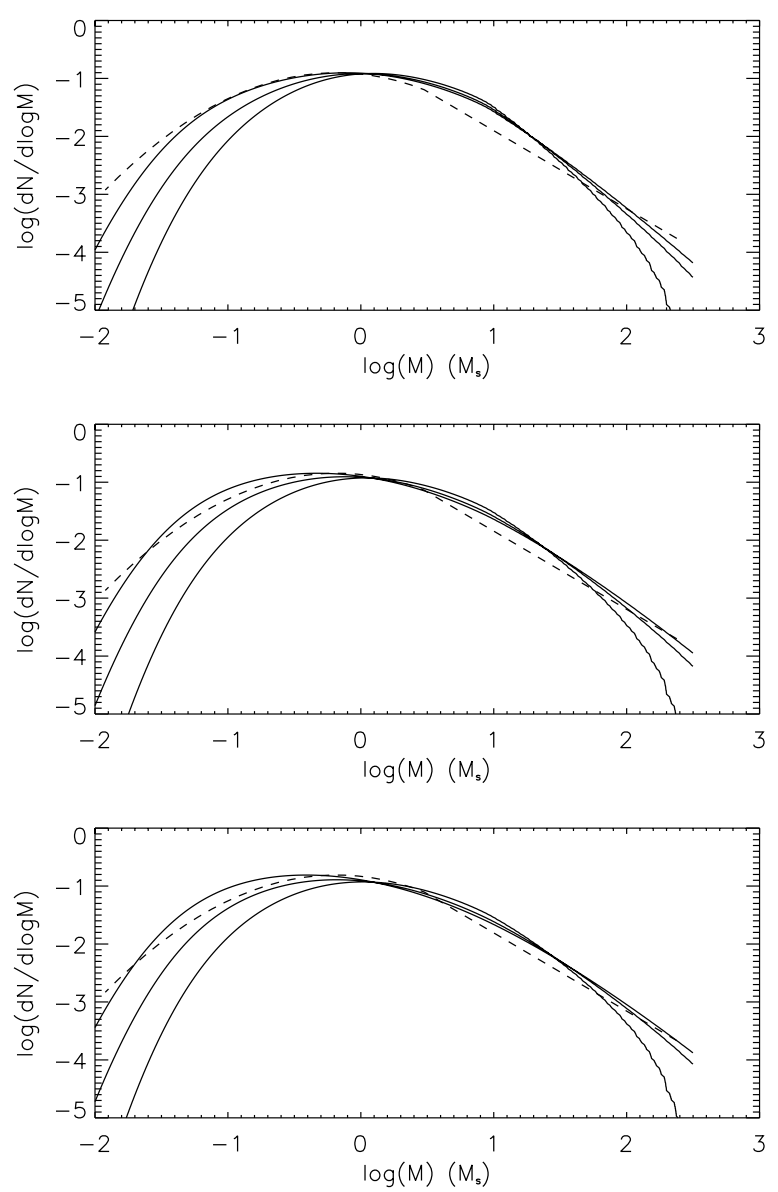

Figure 17. Integrated mass spectrum of self-gravitating objects (prestellar cores) for three values of $\gamma_{\mathrm{cl}}$, the index of the mass spectrum of the star-forming clumps. Top panel: $\gamma_{\mathrm{cl}}=2$; middle panel: $\gamma_{\mathrm{cl}}=1.7$; bottom panel: $\gamma_{\mathrm{cl}}=1.5$. The three solid curves correspond to three different values for the upper value of the accretion rate, namely (from the leftmost line to the rightmost one), $\dot{M}_{\text {sup }}=$ $10^{-1}, 10^{-2}$, and $10^{-3} M_{\odot} \mathrm{yr}^{-1}$. The dashed line is the SCIMF.

For $\gamma_{\mathrm{cl}}=1.5$ and $\gamma_{\mathrm{cl}}=1.7$, the mass spectra are pretty similar to the results obtained in the previous section for a single clump. The reason is that most of the mass is contained in the most massive clumps. Interestingly, varying the upper value of the accretion rate by two orders of magnitude has only a modest impact on the upper mass part of the total mass spectrum, while below about the mean Jeans mass, the lowmass part of the distribution quantitatively varies by orders of magnitude, the number of low-mass objects, in particular brown dwarfs, strongly decrease with decreasing accretion rate. Values of $\dot{M}_{\text {sup }} \lesssim 10^{-3} M_{\odot} \mathrm{yr}^{-1}$ yield a strong deficit of brown dwarfs compared with the SCIMF, representative of the observed population. As mentioned above, this stems from the smaller cloud size, and thus the smaller Mach number with decreasing accretion rate, yielding a lack of overdense smallscale structures, which are the progenitors of the brown dwarfs (see Paper I and Paper II).

For $\gamma_{\mathrm{cl}}=2$, the difference with the mass spectrum obtained for a single clump is more pronounced. In particular, at intermediate mass, the distribution tends to deviate from the SCIMF. This is due to the mass of the gas being equally distributed between small and large clumps. As the CMF that results from small clumps peaks at larger masses than the CMF produced in massive clumps, this tends to create a small bump at intermediate masses $\left(M \simeq 10 M_{\odot}\right)$. 


\section{CONCLUSION: A PARADIGM FOR STAR FORMATION AND THE INITIAL MASS FUNCTION}

\subsection{Summary of the Results}

In this paper, we have generalized our previous formalism and developed an analytical time-dependent theory of the stellar IMF. Although not very different from the time-independent prestellar mass spectra for given sets of cloud initial conditions (temperature, density, Mach number), the time-dependent ones are not quite identical to the former ones. The most significant differences are a slightly steeper slope at large masses, which arises from the time dependence of the characteristic Jeans mass, and the position of the peak of the CMF, which is shifted toward smaller masses by a factor of the order of $2-3$, depending on the initial conditions. This is a direct consequence of the small-scale structures being rejuvenated several times during the collapsing process of the larger ones. For all explored typical clump conditions, the resulting CMF, while slightly too narrow when considering an isothermal eos, agrees very well with the shifted Chabrier system IMF (i.e., the Chabrier IMF shifted by a factor of $\simeq 3$ to take into account the core-to-star mass conversion efficiency during the collapse) when including the thermodynamics of the gas, confirming the results obtained for the time-independent theory.

This time-dependent theory enables us to derive an expression for the SFR for molecular cloud conditions. Confrontations with previously published SFR theories show some similar trends but quantitative or even qualitative differences. As mentioned in $\mathrm{HC} 11$, these differences arise essentially from the two essential characteristics of our theory, namely, (1) each overdense region dominated by gravity collapses under its own density-dependent dynamical time and (2) there is no particular threshold density/scale for star formation; instead, any overdense region produced by initial density fluctuations is susceptible to collapse, no matter its degree of internal turbulence, if dominated by gravity.

We explore the dependence of the SFR upon the clump properties, namely, the size, the level of turbulence (through the virial parameter), and the magnetic field. We show that the exact value of the SFR depends significantly on these clump parameters, which, unfortunately, are ill-defined quantities, hampering precise theoretical estimates of the SFR in a clump/cloud, in particular, for magnetized clouds. We show that when time dependence is taken into account in the formation of collapsing dense cores, turbulence globally favors star formation, in contrast to the result obtained with the time-independent theory, in agreement with the results of PN. We also show that when the clump is unbound, i.e., $\alpha_{\text {vir }} \gtrsim 1$, the SFR decreases drastically, as the dominant contribution of kinetic (thermal+turbulent) over gravitational energy prevents gravitational collapse of the overdense structures, inhibiting star formation. The magnetic field also reduces the SFR when it is strong enough. Its exact influence depends on the magnetic-density relation and also on its influence on the density PDF, none of these properties being known with great accuracy.

Our calculations show that SFR correlates with both the gas surface density of the surrounding region and the size of the clump, and occurs dominantly in dense and/or massive-thus bound-clumps. Such large clumps and dense regions, however, represent only a modest fraction $(\lesssim 20 \%)$ of typical clouds. In contrast, for the smallest clumps, the SFR decreases drastically below a typical density $\Sigma_{g} \approx 100 M_{\odot} \mathrm{pc}^{-2}$, i.e., a volume density $\bar{n} \approx 2500\left(R_{c} / 1 \mathrm{pc}\right)^{-1} \mathrm{~cm}^{-3}$, i.e., a visual extinction
$A_{V} \approx 7$, in excellent agreement with observational determinations in Milky Way molecular clouds. For clumps below about this density, star formation is still taking place, although at a much lower rate, only in very large ( $\gtrsim 10 \mathrm{pc}$ ) clumps, which are very rare. This stresses the need to explore large fields of view to accurately determine the SFR in low-density regions. We stress that this abrupt change in the SFR does not stem from an arbitrarily defined threshold for star formation, but naturally arises from the theory and reflects the fact that pc-size, low-density clumps barely form stars essentially because of their too large characteristic Jeans length compared with their size and their too low level of turbulence.

Interestingly enough, the aforementioned density corresponds to the density above which the cold gas in the ISM becomes essentially composed of molecular hydrogen, $\mathrm{H}_{2}$. Indeed, simulations of compressible turbulence coupled with hydrogen chemistry (Glover \& Mac Low 2007, see also Krumholz et al. 2009) suggest that (1) $\mathrm{H}_{2}$ forms much more efficiently in (compressible) turbulent gas than in quiescent gas owing to the much shorter formation timescale and that (2) above $n \sim 300 \mathrm{~cm}^{-3}$, the $\mathrm{H}_{2}$ formation rate, which scales as the square of the number density, $n^{2}$, becomes larger than the photodissociation rate, allowing the efficient in situ formation of molecular hydrogen. The gas becomes dominantly (entirely) molecular above about $5000 \mathrm{~cm}^{-3}\left(10^{4} \mathrm{~cm}^{-3}\right)$ and remains "trapped" and shielded from external UV radiation in the collapsing structures. Our calculations thus support Glover \& Mac Low's (2007) suggestion that dense (bound or unbound) star-forming regions drive $\mathrm{H}_{2}$ formation, and not the opposite. Phrased differently, the formation of molecular hydrogen is not a necessary condition for star formation but instead is a consequence of efficient star formation.

Finally, we derive a fully time dependent calculation of the core mass spectrum by considering a simple time-dependent clump model. That is, instead of assuming fixed clump conditions (density, mass, size), we consider an evolving, accreting clump assuming a constant accretion rate. In order to explore the parameter space, we have conducted calculations for various accretion rates, typical of the observed determinations, assuming that the clump parameters obey the standard Larson scaling relations. The resulting core mass spectrum remains in very good agreement with the observationally derived distribution and reproduces various behaviors observed in simulations of converging flows. We also investigate the core mass spectrum that results from the contribution of a clump distribution such that $d N_{c} / d M_{c} \propto M^{-1.7}$. We show that the resulting core mass spectrum is dominated by the mass spectrum of the biggest clumps because they contain most of the mass.

Our SFR model provides the general framework that includes the simplified model suggested by Lada et al. (2012), which relies on the fraction of dense (molecular) gas in the cloud and thus critically depends on a threshold density. As mentioned above (and shown in Figure 7), our theory naturally predicts a strong, roughly quadratic correlation between the SFR and the gas density, predicted to become dominantly molecular above $\sim 5000 \mathrm{~cm}^{-3}$. As shown in Section 4.3.1, such a correlation immediately implies a similar correlation with the mass of the clump/cloud itself, i.e., with the mass of molecular hydrogen above the aforementioned value. The model suggested by Lada et al. (2012) is thus a direct consequence of our general theory of the IMF+SFR. As mentioned above, however, star formation is still predicted to take place, although at a much lower rate, in large, low-density clouds, which will be composed essentially of atomic hydrogen. 
However, in contrast to what has often been claimed in the literature, we show that there is no universal value of the SFR, and that this latter does not simply correlate linearly with gas density. Indeed, as mentioned above, the SFR strongly depends not only on the gas density but also on the clump mass/size, which leads to a large scatter in SFR values.

\subsection{A Paradigm for Star Formation and for the Theory of the IMF}

The analytical theory described in our previous and present papers, which correctly reproduces various observational constraints, as discussed in these papers, suggests the following paradigm for star formation and for the resulting CMF/IMF.

1. Compressible (shock dominated) large-scale turbulence in the cloud, due to various possible mechanisms, such as accretion, converging cold and warm flows, or star formation itself, generates a field of density fluctuations at all scales in the cloud. The (nearly lognormal) PDF of these fluctuations is entirely determined by the characteristic (universal) log-density power spectrum index of turbulence. This PDF leads to overdense regions that correspond to the observed clump mass spectrum (see Paper I). Observations suggest that the clumps have a filamentary structure. As they accrete mass and dissipate kinetic energy, the densest clumps become gravitationally unstable above a typical density $\bar{N}_{\mathrm{H}_{2}} \approx 10^{22} \mathrm{~cm}^{-2}$ (i.e., $\bar{n} \approx 10^{4}\left(R_{c} / 1 \mathrm{pc}\right)^{-1} \mathrm{~cm}^{-3}$ for a spherical clump) for a temperature $T \sim 10 \mathrm{~K}$, triggering the fragmentation into prestellar cores. Although probably quantitatively affecting our results by numerical factors of the order of unity, this filamentary nature of the clumps does not modify the general framework of our theory, which indeed predicts a very narrow CMF below about this density for pc-size clumps, yielding a negligible number of prestellar cores.

2. Fragmentation then introduces a scale dependence (and thus a density dependence) in the process and sets up the virial criterion for gravitational collapse (see Paper I). As demonstrated in Paper I and in Chabrier \& Hennebelle (2011), we suggest that turbulence plays a crucial role in setting the massive initial mass reservoir distribution (and thus the Salpeter slope of the IMF), progenitors of massive stars, above about the mean thermal Jeans mass, at the early stages of star formation, not by providing a pressure support, in a static sense, but by dispersing the gas within these structures, which would otherwise have collapsed, until they reach a mass that we identify as their turbulent Jeans mass (see Equation (28)).

3. Below about the typical density mentioned above, our theory predicts a drastically decreasing $\mathrm{CMF}$, and thus SFR, for the typical size of the dominant clump population, essentially because the characteristic Jeans length of the clump becomes comparable to or larger than its size, stabilizing the clump against fragmentation. For a filament, the same density corresponds to the critical mass per unit length below which the clump is gravitationally stable, yielding the same conclusion. We predict, however, that star formation can still occur below this density in very large ( $\gtrsim 10)$ pc size) clumps, large enough to significantly exceed their typical Jeans length and generate large enough turbulence levels. This seems to be supported by the observed population of Class II cores in low-density, large clumps (Gutermuth et al. 2011) and stresses the need to observe very large areas at low density in order to get statistically significant core detections. Such clumps, however, are very seldom, as inferred from the steeply decreasing clump size spectrum, and the probability of finding prestellar cores in low-density environments drops drastically, making their detection very difficult.

4. Star formation is thus a continuous process and can occur, statistically speaking, in any density environment, as any turbulence-induced overdense region can collapse, if dominated by gravity, and eventually produce a prestellar core. There is thus no real "threshold" for star formation. This leads to a direct correspondence between the SFR and the gas (atomic + molecular) density, as observed and indeed predicted by the theory, with star formation occurring most actively in the densest regions of molecular clouds, which entails only a small $(\lesssim 20 \%)$ fraction of their mass. However, as mentioned above, star formation is basically choked off below $\bar{n} \sim 1000 \mathrm{~cm}^{-3}$ for the typical $(\sim$ pc) size of most clumps. Above this density, the $\mathrm{H}_{2}$ formation rate, which increases as the square of the gas density, dominates the photodissociation rate and the gas quickly becomes dominantly and entirely molecular. This drastically decreases the photoelectric heating efficiency, causing in turn a drop in dust and gas temperature (Tielens \& Hollenbach 1985; Glover \& Mac Low 2007). Star formation thus promotes $\mathrm{H}_{2}$ formation, and not the opposite.

We stress, however, that from the general point of view, star formation strongly depends on the clump characteristic properties (mass, size); therefore, there is no "universal" relation between SFR and gas density, but instead large variations depending on the clump's environment.

This paradigm, which relies on the results presented in the present and former papers, shows that an SFR determined at the early stages by turbulence-induced fluctuations provides quite a consistent picture of star formation in Milky Way molecular clouds, with star formation occurring dominantly in the densest regions of the cloud. This gravo-turbulent picture of star formation correctly predicts the observed CMF over the entire mass range from brown dwarfs to massive stars and naturally leads to an SFR versus clump masses/sizes and gas density correlations in very good agreement with observational determinations. We stress, however, that ill-defined quantities such as the size of the clump or the dynamical efficiency of core-to-star mass conversion, which may well vary from clump to clump, make the determination of the SFR uncertain by a factor of a few.

We thank the anonymous referee for helpful comments and Christoph Federrath for a critical reading of the manuscript. The research leading to these results has received funding from the European Research Council under the European Community's Seventh Framework Programme (FP7/2007-2013 Grant Agreement No. 247060). This work was initiated while G.C. was a visitor of the Max Planck Institute for Astrophysics, where he benefited from numerous discussions with various colleagues.

\section{REFERENCES}

André, P., Men'shchikov, A., Bontemps, S., et al. 2010, A\&A, 518, L102 André, P., Men'sikov, A., Koenyeces, V., et al. 2011, in Stellar Clusters \& Associations: A RIA Workshop on Gaia, ed. E. Alfaro \& T. Gallego (Granada, Spain: IAA-CSIC), 321

Arzoumanian, D., André, P., Didelon, P., et al. 2011, A\&A, 529, L6

Audit, E., \& Hennebelle, P. 2010, A\&A, 511, 76 
Banerjee, R., Vázquez-Semadeni, E., Hennebelle, P., \& Klessen, R. 2009, MNRAS, 398, 1082

Bate, M. 2009, MNRAS, 392, 1363

Beresnyak, A., Lazarian, A., \& Cho, J. 2005, ApJL, 624, L93

Bontemps, S., Motte, F., Csengeri, T., \& Schneider, N. 2010, A\&A, 524, 18

Bressert, E., Bastian, N., Gutermuth, R., et al. 2010, MNRAS, 409, L54

Brunt, C., Federrath, C., \& Price, D. 2010, MNRAS, 405, L56

Chabrier, G. 2003, PASP, 115, 763

Chabrier, G., \& Hennebelle, P. 2010, ApJL, 725, L79

Chabrier, G., \& Hennebelle, P. 2011, A\&A, 534, 106

Ciardi, A., \& Hennebelle, P. 2010, MNRAS, 409, L39

Clark, P., Klessen, R., \& Bonell, I. 2007, MNRAS, 379, 57

Commercon, B., Hennebelle, P., Audit, E., Chabrier, G., \& Teyssier, R. 2010, A\&A, 510, L3

Commercon, B., Hennebelle, P., \& Henning, T. 2011, ApJL, 742, L9

Crutcher, R. 1999, ApJ, 520, 706

Crutcher, R., Wandelt, B., Heiles, C., Falgarone, E., \& Troland, T. 2010, ApJ, 725,466

Draine, B. 2003, ApJ, 598, 1017

Enoch, L., Glenn, J., Evans, N., et al. 2007, ApJ, 666, 982

Evans, N., Dunham, M., Jorgensen, J., et al. 2009, ApJS, 181, 321

Falgarone, E., Hily-Blant, P., \& Levrier, F. 2004, Ap\&SS, 292, 285

Federrath, C., \& Klessen, R. 2012, ApJ, 761, 156

Federrath, C., Klessen, R., \& Schmidt, W. 2008, ApJ, 688, 79

Federrath, C., Roman-Duval, J., Klessen, R., Schmidt, W., \& MacLow, M.-M. 2010, A\&A, 512, 81

Fiege, J., \& Pudritz, R. 2000, MNRAS, 311, 85

Fukui, Y., Kawamura, A., Wong, T., et al. 2009, ApJ, 705, 144

Glover, S., \& Mac Low, M. 2007, ApJS, 169, 239

Gutermuth, R. A., Pipher, J. L., Megeath, S. T., et al. 2011, ApJ, 739, 84

Hatchell, J., Richer, J., Fuller, G., et al. 2005, A\&A, 440, 151

Heiderman, A., Evans, N., Allen, L., Huard, T., \& Heyer, M. 2010, ApJ, 723, 1019

Heithausen, A., Bensch, F., Stutzki, J., Falgarone, E., \& Panis, J.-F. 1998, A\&A, 331, L65

Heitsch, F., Hartmann, L., Slyz, A., Devriendt, J., \& Burkert, A. 2008, ApJ, 674,316

Hennebelle, P. 2012, A\&A, 545, 147

Hennebelle, P., Banerjee, R., Vázquez-Semadeni, E., Klessen, R., \& Audit, E. 2008, A\&A, 486, L43

Hennebelle, P., \& Chabrier, G. 2008, ApJ, 684, 395 (Paper I)

Hennebelle, P., \& Chabrier, G. 2009, ApJ, 702, 1428 (Paper II)

Hennebelle, P., \& Chabrier, G. 2011a, IAUS, 270, 159

Hennebelle, P., \& Chabrier, G. 2011b, ApJL, 743, L29

Hennebelle, P., Commercon, B., Joos, M., et al. 2011, A\&A, 528, 72

Hennebelle, P., \& Teyssier, R. 2008, A\&A, 477, 25

Heyer, M., Krawczyk, C., Duval, J., \& Jackson, J. 2009, ApJ, 699, 1092

Hill, T., Motte, F., Didelon, P., et al. 2011, A\&A, 533, 94

Hopkins, P. 2012a, MNRAS, 423, 2016

Hopkins, P. 2012b, MNRAS, 423, 2037

Inutsuka, S.-I., \& Miyama, S. 1992, ApJ, 388, 392

Inutsuka, S.-I., \& Miyama, S. 1997, ApJ, 480, 681

Jedamzik, K. 1995, ApJ, 448, 1

Johnstone, D., Di Francesco, J., \& Kirk, H. 2004, ApJL, 611, L45

Kainulainen, J., Beuther, H., Banerjee, R., Federrath, C., \& Henning, T. 2011, A\&A, 530, 64

Kainulainen, J., Beuther, H., Henning, T., \& Plume, R. 2009, A\&A, 508, L35
Kawamura, A., Mizuno, Y., Minamidani, T., et al. 2009, ApJS, 184, 1

Kennicutt, R., \& Evans, N. 2012, ARA\&A, 50, 531

Kramer, C., Stutzki, J., Rohrig, R., \& Corneliussen, U. 1998, A\&A, 329, 249

Kritsuk, A., Norman, M., Padoan, P., \& Wagner, R. 2007, ApJ, 665, 416

Krumholz, M., Dekel, A., \& McKee, C. 2012, ApJ, 745, 69

Krumholz, M., Klein, R., \& McKee, C. 2007, ApJ, 656, 959

Krumholz, M., \& McKee, C. 2005, ApJ, 630, 250 (KMK)

Krumholz, M., McKee, C., \& Tumlinson, J. 2009, ApJ, 693, 216

Lada, C., Forbrich, J., Lombardi, M., \& Alves, J. 2012, ApJ, 745, 190

Lada, C., Lombardi, M., \& Alves, J. 2010, ApJ, 724, 687

Larson, R. 1981, MNRAS, 194, 809

Larson, R. 1985, MNRAS, 214, 379

Larson, R. 2003, RPPh, 66, 1651

Liu, G., Koda, J., Calzetti, D., Fukuhara, M., \& Momose, R. 2011, ApJ, 735,63

Longmore, S., Pillai, T., Keto, E., Zhang, Q., \& Qiu, K. 2011, ApJ, 726, 97

Machida, M., Matsumoto, T., Hanawa, T., \& Tomisaka, K. 2005, MNRAS, 362,382

Machida, M., Tomisaka, K., Matsumoto, T., \& Inutsuka, S.-I. 2008, ApJ, 677,327

MacLow, M.-M., \& Klessen, R. 2004, RvMP, 76, 125

Matzner, C. D., \& McKee, C. 2000, ApJ, 545, 364

Maury, A., André, P., Men'shchikov, A., Könyves, V., \& Bontemps, S. 2011, A\&A, 535, 77

Men'shchikov, A., André, Ph., Didelon, P., et al. 2010, A\&A, 518, L103

Molina, F., Glover, S., Federrath, C., \& Klessen, R. 2012, MNRAS, 423, 2680

Molinari, S., Swinyard, B., Bally, J., et al. 2010, A\&A, 518, L100

Murray, N. 2011, ApJ, 729, 133

Nakamura, F., \& Li, Z.-Y. 2008, ApJ, 687, 354

Onishi, T., Mizuno, A., Kawamura, A., et al. 1998, ApJ, 502, 296

Ostriker, E., McKee, C., \& Leroy, A. 2010, ApJ, 721, 975

Ostriker, J. 1964, ApJ, 140, 1056

Padoan, P., Juvela, M., Goodman, A., \& Nordlund, A. 2001, ApJ, 553, 227

Padoan, P., \& Nordlund, A. 1999, MNRAS, 526, 279

Padoan, P., \& Nordlund, A. 2011, ApJ, 730, 40 (PN)

Padoan, P., Nordlund, A., \& Jones, B. 1997, MNRAS, 288, 145

Palau, A., Fuente, A., Girart, J., et al. 2013, ApJ, 762, 120

Passot, T., \& Vázquez-Semadeni, E. 1998, PhRvE, 58, 4501

Peretto, N., \& Fuller, G. 2010, ApJ, 723, 555

Press, W., \& Schechter, P. 1974, ApJ, 187, 425

Price, D., \& Bate, M. 2008, MNRAS, 385, 1820

Renaud, F., Kraljic, K., \& Bournaud, F. 2012, ApJL, 760, L16

Schmidt, W., Federrath, C., Hupp, M., Kern, S., \& Niemeyer, J. 2009, A\&A, 494, 127

Schmidt, W., Kern, S., Federrathn, C., \& Klessen, R. 2010, A\&A, 516, 25

Smith, R., Longmore, S., \& Bonnell, I. 2009, MNRAS, 400, 1775

Tielens, A., \& Hollenbach, D. 1985, ApJ, 291, 722

Troland, T., \& Heiles, C. 1986, ApJ, 301, 339

Vázquez-Semadeni, E. 1994, ApJ, 423, 681

Vázquez-Semadeni, E., Ballesteros-Paredes, J., \& Klessen, R. 2003, ApJL, $585, \mathrm{~L} 131$

Vázquez-Semadeni, E., Gómez, G., Jappsen, K., Gonzalez, R. G., \& Klessen, R. 2007, ApJ, 657, 870

Ward-Thompson, D., Kirk, J., André, P., et al. 2010, A\&A, 518, L92

Yano, T., Nagashima, M., \& Gouda, N. 1996, ApJ, 466, 1

Zamora-Avilés, M., Vázquez-Semadeni, E., \& Colín, P. 2012, ApJ, 751, 77 